\title{
Report on the Absolute Differential Equations I
}

\author{
Veronika Chrastinová ${ }^{1}$ and Václav Tryhuk ${ }^{2 *}$ \\ ${ }^{1}$ Brno University of Technology, Faculty of Civil Engineering, Department of Mathematics, \\ Veveří 331/95, 60200 Brno, Czech Republic \\ Email: chrastinova.v@fe.vutbr.cz \\ ${ }^{2}$ Brno University of Technology, Faculty of Civil Engineering, AdMaS centre, Purkyňova 139, \\ 61200 Brno, Czech Republic \\ Email: tryhuk.v@fce.vutbr.cz, tryhuk.v@outlook.com
}

\begin{abstract}
The article provides a survey of the absolute theory of general systems of (partial) differential equations. The equations are relieved of all additional structures and subject to quite arbitrary change of the variables. An abstract mathematical theory in the Bourbaki sense with its own concepts and technical tools follows. In particular the external, internal, generalized and higher-order symmetries and infinitesimal symmetries together with the E. Cartan's prolongations, various characteristics, the involutivity and the controllability structures are clarified in genuinely coordinate-free terms without any use of the common jet mechanisms.
\end{abstract}

Keywords: Higher-order transformations; symmetry; diffiety; involutivity; controllability; characteristics.

\section{Preface}

We motivate the central concepts and informally describe the main task in order to give some impressions on our subject, however, this is not a logical prerequisite for the text to follow.

\subsection{The Higher-order Transformations (the Morphisms)}

Let us recall the jet coordinates

$$
x_{i}, w_{I}^{j} \quad\left(j=1, \ldots, m ; I=i_{1} \cdots i_{r} ; i, i_{1}, \ldots, i_{r}=1, \ldots, n ; r=0,1, \ldots\right)
$$

where $x_{i}$ are independent variables, $w^{j}$ (empty $I$ ) dependent variables and $w_{I}^{j}$ (nonempty $I$ ) correspond to the derivatives

$$
w_{I}^{j}=\frac{\partial w^{j}}{\partial x_{I}}=\frac{\partial^{r} w^{j}}{\partial x_{i_{1}} \cdots \partial x_{i_{r}}} \quad\left(I=i_{1} \cdots i_{r}\right) .
$$

Let us moreover introduce the equations

$$
\bar{x}_{i}=X_{i}\left(\cdot \cdot, x_{i^{\prime}}, w_{I^{\prime}}^{j^{\prime}}, \cdot \cdot\right), \bar{w}^{j}=W^{j}\left(\cdot \cdot, x_{i^{\prime}}, w_{I^{\prime}}^{j^{\prime}}, \cdot .\right) \quad(i=1, \ldots, n ; j=1, \ldots, m)
$$

where $X_{i}$ and $W^{j}$ are given functions of a finite number of variables (1). They are interpreted as the transformation formulae: the functions

$$
w^{j}=w^{j}\left(x_{1}, \ldots, x_{n}\right) ; j=1, \ldots, m ;
$$

are transformed into certain functions

$$
\bar{w}^{j}=\bar{w}^{j}\left(\bar{x}_{1}, \ldots, \bar{x}_{n}\right) ; j=1, \ldots, m ;
$$

and this is made as follows. Denoting

$$
\mathcal{X}_{i}=X_{i}\left(\cdot \cdot x_{i^{\prime}}, \frac{\partial w^{j^{\prime}}}{\partial x_{I^{\prime}}}\left(x_{1}, \ldots, x_{n}\right), \cdot \cdot\right)=\mathcal{X}_{i}\left(x_{1}, \ldots, x_{n}\right) \quad(i=1, \ldots, n),
$$


we suppose that

$$
\operatorname{det}\left(\frac{\partial \mathcal{X}_{i^{\prime}}}{\partial x_{i}}\right)=\operatorname{det}\left(D_{i} \mathcal{X}_{i^{\prime}}\right) \neq 0 \quad\left(D_{i}=\frac{\partial}{\partial x_{i}}+\sum w_{I i}^{j} \frac{\partial}{\partial w_{I}^{j}}\right) .
$$

Then the implicit system $\bar{x}_{i}=\mathcal{X}_{i}\left(x_{1}, \ldots, x_{n}\right) ; i=1, \ldots, n$; admits a solution $x_{i}=\overline{\mathcal{X}}_{i}\left(\bar{x}_{1}, \ldots, \bar{x}_{n}\right)$; $i=1, \ldots, n$; and provides the desired result

$$
\bar{w}^{j}=W^{j}\left(\cdot \cdot \overline{\mathcal{X}}_{i}, \frac{\partial w^{j^{\prime}}}{\partial x_{I^{\prime}}}\left(\overline{\mathcal{X}}_{1}, \ldots, \overline{\mathcal{X}}_{n}\right), \cdot \cdot\right)=\bar{w}^{j}\left(\bar{x}_{1}, \ldots, \bar{x}_{n}\right) \quad(j=1, \ldots, m) .
$$

One can also obtain the transformation of the derivatives

$$
\bar{w}_{I}^{j}=\frac{\partial \bar{w}^{j}}{\partial \bar{x}_{I}}=W_{I}^{j}\left(\cdot \cdot, x_{i^{\prime}}, w_{I^{\prime}}^{j^{\prime}}, \cdot \cdot\right) \quad(\text { all } j \text { and } I)
$$

which complete the equations (2). They satisfy the recurrence

$$
\sum W_{I i}^{j} D_{i} X_{i^{\prime}}=D_{i^{\prime}} W_{I}^{j} \quad\left(\text { all } i^{\prime}, j, I\right)
$$

and we altogether speak of a morphism (2), (3). If there exists the inverse morphism

$$
x_{i}=\bar{X}_{i}\left(\cdot \cdot \bar{x}_{i^{\prime}}, \bar{w}_{I^{\prime}}^{j^{\prime}}, \cdot \cdot\right), w_{I}^{j}=\bar{W}_{I}^{j}\left(\cdot \cdot \bar{x}_{i^{\prime}}, \bar{w}_{I^{\prime}}^{j^{\prime}}, \cdot \cdot\right),
$$

we speak of an automorphism (or: symmetry). The structure of the totality of all symmetries unexpectedly manifests as an unheard-of mystery [1],[2]. Just the symmetries are important since they produce the higher-order equivalences of differential equations.

\subsection{Example: the Wave Construction}

Assuming $n=1$, we abbreviate

$$
x=x_{1}, \bar{x}=\bar{x}_{1}, w_{r}^{j}=w_{1 \cdots 1}^{j}, \bar{w}_{r}^{j}=\bar{w}_{1 \cdots 1}^{j} \quad(r \text { terms }) .
$$

Let $V=V\left(x, w_{0}^{1}, \ldots, w_{0}^{m}, \bar{x}, \bar{w}_{0}^{1}, \ldots, \bar{w}_{0}^{m}\right)$ be a given function.

Proposition [3],[4]. If the implicit system

$$
V=D V=\cdots=D^{m} V=0 \quad\left(D=\frac{\partial}{\partial x}+\sum w_{r+1}^{j} \frac{\partial}{\partial w_{r}^{j}}\right)
$$

admits a solution

$$
\bar{x}=X\left(x, w_{0}^{1}, \ldots, w_{m}^{m}\right), \bar{w}_{0}^{j}=w_{0}^{j}\left(x, w_{0}^{1}, \ldots, w_{m}^{m}\right) \quad(j=1, \ldots, m)
$$

such that $D X \neq 0$ and the implicit system

$$
V=\bar{D} V=\cdots=\bar{D}^{m} V=0 \quad\left(\bar{D}=\frac{\partial}{\partial \bar{x}}+\sum \bar{w}_{r+1}^{j} \frac{\partial}{\partial \bar{w}_{r}^{j}}\right)
$$

admits a solution

$$
x=\bar{X}\left(\bar{x}, \bar{w}_{0}^{1}, \ldots, \bar{w}_{m}^{m}\right), w_{0}^{j}=\bar{w}_{0}^{j}\left(\bar{x}, \bar{w}_{0}^{1}, \ldots, \bar{w}_{m}^{m}\right) \quad(j=1, \ldots, m)
$$

such that $\bar{D} \bar{X} \neq 0$ then (6) and (7) are symmetric inverse of each other.

The Proposition can be extensively generalized for the case $n>1$ and all the classical Lie contact transformations are involved if $m=1$. Except for this Lie's favourable subcase, all such symmetries destroy the finite-order jet spaces. 


\subsection{The Infinitesimal Modification [5],[6],[7]}

The ancient "linear approximation" of equations (2) and (3) reads

$$
\bar{x}_{i}=x_{i}+\varepsilon z_{i}\left(\cdot \cdot x_{i^{\prime}}, w_{I^{\prime}}^{j^{\prime}}, \cdot \cdot\right), \bar{w}_{I}^{j}=w_{I}^{j}+\varepsilon z_{I}^{j}\left(\cdot \cdot, x_{i^{\prime}}, w_{I^{\prime}}^{j^{\prime}}, \cdot \cdot\right)
$$

where $\varepsilon$ is the famed "small parameter". In the rigorous theory, let us instead introduce the vector field

$$
Z=\sum z_{i} \frac{\partial}{\partial x_{i}}+\sum z_{I}^{j} \frac{\partial}{\partial w_{I}^{j}} \quad\left(z_{I i}^{j}=D_{i} z_{I}^{j}-\sum w_{I i^{\prime}}^{j} D_{i^{\prime}} z_{i}\right)
$$

with the recurrence following from (4).

Warning. In contrast to actual convention, we speak of a variation and the common term the generalized (or: higher-order, or: Lie-Bäcklund) infinitesimal transformation (briefly: the symmetry) is retained only for the case when the vector field $Z$ generates a true (local) Lie group of transformations

$$
\bar{x}_{i}[t]=X_{i}\left(\cdot, x_{i^{\prime}}, w_{i^{\prime}}^{j^{\prime}}, \cdot \cdot ;\right), w_{I}^{j}[t]=W_{I}^{j}\left(\cdot \cdot, x_{i^{\prime}}, w_{i^{\prime}}^{j^{\prime}}, \cdot \cdot t\right) \quad(-\varepsilon<t<\varepsilon)
$$

depending on the group parameter $t$, that is, for the favourable case when the Lie system

$$
\frac{\partial X_{i}}{\partial t}=z_{i}\left(\cdot \cdot, X_{i^{\prime}}, W_{i^{\prime}}^{j^{\prime}}, \cdot \cdot\right), \frac{\partial W_{I}^{j}}{\partial t}=z_{I}^{j}\left(\cdot \cdot, X_{i^{\prime}}, W_{i^{\prime}}^{j^{\prime}}, \cdot \cdot\right) \quad\left(\left.X_{i}\right|_{t=0}=x_{i},\left.W_{I}^{j}\right|_{t=0}=w_{I}^{j}\right)
$$

admits a solution. While all variations are given by simple explicit formula (8), the totality of all infinitesimal symmetries is unknown.

\subsection{Example: Variations and Symmetries}

We again suppose $n=1$. The vector field

$$
D=Z=\frac{\partial}{\partial x}+\sum w_{r+1}^{j} \frac{\partial}{\partial w_{r}^{j}} \quad\left(z_{1}=1, z_{1 \cdots 1}^{j}=z_{r}^{j}=w_{r+1}^{j} \text { with } r \text { terms }\right)
$$

is clearly a variation but not a symmetry since the Lie system

$$
\frac{\partial X}{\partial t}=z_{1}=1, \frac{\partial W_{r}^{j}}{\partial t}=W_{r+1}^{j}=D W_{r}^{j} \quad\left(\left.X\right|_{t=0}=x,\left.W_{r}^{j}\right|_{t=0}=w_{r}^{j}\right)
$$

for the corresponding Lie group is contradictory. On the contrary, the vector field

$$
Z=\sum w_{r+1}^{1} \frac{\partial}{\partial w_{r}^{k}} \quad(m \geq 2 ; \text { the sum over } k=2, \ldots, m \text { and } r=0,1, \ldots)
$$

generates the very simple "higher-order Lie group" of the morphisms

$$
\bar{x}[t]=x, \bar{w}_{r}^{1}[t]=w_{r}^{1}, \bar{w}_{r}^{k}[t]=w_{r}^{k}+t w_{r+1}^{1} \quad(k=2, \ldots, m ; r=0,1, \ldots) .
$$

This very simple group does not preserve many of the classical concepts, even the order of the differential equations.

\subsection{External Differential Equations [7]}

Let us denote $\mathbf{M}(m, n)$ the space with coordinates (1) from now on. (This is the classical jet space without any differential constraints.) Differential equations are traditionally interpreted as the subspace $\mathbf{M} \subset \mathbf{M}(m, n)$ defined by certain equations

$$
D_{i_{1}} \cdots D_{i_{r}} f^{k}=0 \quad\left(k=1, \ldots, K ; i_{1}, \ldots, i_{r}=1, \ldots, n ; r=0,1, \ldots\right)
$$


where $f^{1}, \ldots, f^{K}$ are given functions of variables (1). Together with the "primary" equations $f^{1}=\cdots=$ $f^{K}=0$, also all consequences (9) are taken into account. The compatibility is implicitly contained in the definition.

Let us recall the morphisms. By using the more precise pull-back notation

$$
\mathbf{m}^{*} x_{i}=\bar{x}_{i}, \mathbf{m}^{*} w_{I}^{j}=\bar{w}_{I}^{j}
$$

in the equations (2) and (3), they may be interpreted as a mapping $\mathbf{m}: \mathbf{M}(m, n) \rightarrow \mathbf{M}(m, n)$, the morphism of the space $\mathbf{M}(m, n)$. Assuming moreover $\mathbf{m} \mathbf{M} \subset \mathbf{M}$, then $\mathbf{m}$ is said to be the external morphism of $\mathbf{M}$ (or: of differential equations (9)) and the external automorphism (or: external symmetry) in the invertible case (5).

The infinitesimal concepts are analogous: if the vector field (8) is tangent to $\mathbf{M}$ (and therefore $Z$ is a vector field on $\mathbf{M}$ as well) then $Z$ is called the external variation of $\mathbf{M}$ (or: of differential equations (9)) and if $Z$ moreover generates a group, we have the external infinitesimal symmetry.

\subsection{Internal Differential Equations [7]}

The external morphism of $\mathbf{M}$ whose restriction to $\mathbf{M}$ is invertible is the internal symmetry. The external variation $Z$ of $\mathbf{M}$ which generates a Lie group on $\mathbf{M}$ is the internal infinitesimal symmetry. These internal concepts are in fact independent of the localization $\mathbf{M}$ in the ambient space $\mathbf{M}(m, n)$. The reasons are as follows.

Let $\Omega(m, n)$ be the module of all contact forms

$$
\omega=\sum f_{I}^{j} \omega_{I}^{j} \quad\left(\text { finite sum, } \omega_{I}^{j}=\mathrm{d} w_{I}^{j}-\sum w_{I i}^{j} \mathrm{~d} x_{i}\right)
$$

on the space $\mathbf{M}(m, n)$. One can observe that the recurrence (3) is equivalent to the inclusion $\mathbf{m}^{*} \Omega(m, n) \subset$ $\Omega(m, n)$. Analogously the recurrence in (8) is expressed by the inclusion $\mathcal{L}_{Z} \Omega(m, n) \subset \Omega(m, n)$ for the Lie derivative.

Let $\Omega$ be the restriction of the module $\Omega(m, n)$ to the subspace $\mathbf{M} \subset \mathbf{M}(m, n)$. The morphism $\mathbf{m}$ restricted to $\mathbf{M}$ clearly satisfies $\mathbf{m}^{*} \Omega \subset \Omega$. Analogously the variation $Z$ restricted to $\mathbf{M}$ satisfies the inclusion $\mathcal{L}_{Z} \Omega \subset \Omega$. Therefore both concepts are characterized without the use of the ambient space $\mathbf{M}(m, n)$. However more is true: even the module $\Omega$ itself can be characterized in abstract terms, we speak of a diffiety $\Omega$ on $\mathbf{M}$, see below.

Altogether we obtain the internal theory for the differential equations (9) not affected by the inclusion into the ambient jet space $\mathbf{M}(m, n)$.

\subsection{Example: the Internal Symmetry}

Assuming $n=1$, we introduce the subspace $\mathbf{M} \subset \mathbf{M}(m, 1)$ defined by the equations

$$
D^{r} w_{2}^{j}=w_{r+2}^{j}=0 \quad(j=1, \ldots, m ; r=0,1, \ldots) .
$$

Then the morphism $\mathbf{m}: \mathbf{M}(m, 1) \rightarrow \mathbf{M}(m, 1)$ where

$$
\mathbf{m}^{*} x=\bar{x}=x, \mathbf{m}^{*} w_{r}^{j}=\bar{w}_{r}^{j}=w_{r}^{j}+w_{r+1}^{j} \quad(j=1, \ldots, m ; r=0,1, \ldots)
$$

is clearly noninvertible but the restriction to $\mathbf{M}$

$$
\mathbf{m}^{*} x=x, \mathbf{m}^{*} w_{0}^{j}=w_{0}^{j}+w_{1}^{j}, \mathbf{m}^{*} w_{1}^{j}=w_{1}^{j} \quad(j=1, \ldots, m)
$$

is a symmetry. Analogously the vector field $Z=D$ is a mere variation on $\mathbf{M}(m, 1)$ but generates the Lie group

$$
\bar{x}[t]=x, \bar{w}_{0}^{j}[t]=w_{0}^{j}+t w_{1}^{j}, \bar{w}_{1}^{j}[t]=w_{1}^{j} \quad(j=1, \ldots, m)
$$

on the space $\mathbf{M}$. So we have the internal but not the external symmetries. 


\subsection{Use of the Pfaffian Equations}

For instance, let us mention the equation

$$
\mathrm{d} z=p \mathrm{~d} x+F(x, y, z, p) \mathrm{d} y
$$

where $x, y, z, p$ may be regarded as the coordinates of the underlying space. The solutions $z=z(x, y)$ parametrized with $x, y$ satisfy

$$
\frac{\partial z}{\partial y}=F\left(x, y, z, \frac{\partial z}{\partial x}\right)
$$

However the same Pfaffian equation

$$
\mathrm{d} \bar{z}=-x \mathrm{~d} p+F(x, y, \bar{z}+p x, p) \mathrm{d} y \quad(\bar{z}=z-p x)
$$

admits the solutions $\bar{z}=\bar{z}(p, y)$ which satisfy

$$
\frac{\partial \bar{z}}{\partial y}=F\left(-\frac{\partial \bar{z}}{\partial p}, y, \bar{z}-p \frac{\partial \bar{z}}{\partial p}, p\right) .
$$

And quite analogously, the same Pfaffian equation

$$
\mathrm{d} y=\frac{1}{F} \mathrm{~d} z-\frac{p}{F} \mathrm{~d} x \quad\left(F \neq 0, F_{p} \neq 0\right)
$$

admits the solutions $y=y(z, x)$ which satisfy

$$
\frac{\partial y}{\partial x}=-p \frac{\partial y}{\partial z} \quad\left(F(x, y, z, p)=1 / \frac{\partial y}{\partial z} \text { determines } p=p\left(x, y, z, \frac{\partial y}{\partial z}\right)\right) .
$$

We conclude. A Pfaffian equation represents many formally quite dissimilar but in fact equivalent differential equations according to the additional choice of the dependent and the independent variables. It follows that a coordinate-free theory should be expressed in terms of the Pfaffian equations.

\subsection{Towards the Diffieties}

Let us finally recall the subspace $\mathbf{i}: \mathbf{M} \subset \mathbf{M}(m, n)$ in the more precise pull-back notation. Then the module $\Omega=\mathbf{i}^{*} \Omega(m, n)$ is interpreted as the restriction of the module of all contact forms to the subspace $\mathbf{M}$ of the jet space $\mathbf{M}(m, n)$. The definition equations (9) imply that vector fields $D_{1}, \ldots, D_{n}$ are tangent to the subspace $\mathbf{M}$ and therefore may be regarded as vector fields on $\mathbf{M}$ as well. They satisfy the crucial identity

$$
\mathcal{L}_{D_{i^{\prime}}} \omega_{I}^{j}=\mathcal{L}_{D_{i^{\prime}}}\left(\mathrm{d} w_{I}^{j}-\sum w_{I i}^{j} \mathrm{~d} x_{i}\right)=\mathrm{d} w_{I i^{\prime}}^{j}-\sum w_{I i i^{\prime}}^{j} \mathrm{~d} x_{i}=\omega_{I i^{\prime}}^{j}
$$

which is clearly true even for the restrictions $\mathbf{i}^{*} \omega_{I}^{j}$ of the forms $\omega_{I}^{j}$ to the space M. Let $\Omega(m, n)_{l} \subset \Omega(m, n)$ be the submodule of all contact forms of the order $l$ at most, hence $|I|=r \leq l$ in all summands (9). Obviously

$$
\Omega(m, n)_{l}+\sum \mathcal{L}_{D_{i^{\prime}}} \Omega(m, n)_{l}=\Omega(m, n)_{l+1} \quad(\text { all } l)
$$

and this property is true even for the restrictions to $\mathbf{M}$, i.e., for the submodules

$$
\Omega_{l}=\mathbf{i}^{*} \Omega(m, n)_{l} \subset \mathbf{i}^{*} \Omega(m, n)=\Omega .
$$

We have in fact discovered the crucial property of the diffieties $\Omega$, see below.

We conclude. Differential equations should be represented by the Pfaffian system $\omega=0(\omega \in \Omega)$ on the space $\mathbf{M}$ where the modules $\Omega$ are described in abstract terms (the internal theory) by using only the property (12). Then the actual choice of the dependent and the independent variables is irrelevant (the absolute approach) in the sense that the quite general formulae (3) are admitted. 


\section{Fundamental Concepts}

We deal with smooth and local category of manifolds and mappings. Our notational convention for a mapping $\mathbf{m}: \mathbf{M} \rightarrow \overline{\mathbf{M}}$ of manifolds allows the definition domain to be a proper open subset of $\mathbf{M}$. Then the parade of the primary concepts denoted by (I)-(VI) looks as follows.

\section{(I) On the manifolds [5]}

Besides the occasional use of the common finite-dimensional spaces, we mainly deal with manifolds $\mathbf{M}$ modelled on $\mathbb{R}^{\infty}$, i.e., there are coordinates $h^{j}: \mathbf{M} \rightarrow \mathbb{R}(j=1,2, \ldots)$ such that the ring $\mathcal{F}(=\mathcal{F}(\mathbf{M})$, the abbreviation whenever possible) of admissible functions $f: \mathbf{M} \rightarrow \mathbb{R}$ involves just the (smooth) composite functions $f=F\left(h^{1}, \ldots, h^{m(f)}\right)$. Then the $\mathcal{F}$-module $\Phi(=\Phi(\mathbf{M}))$ of differential forms $\varphi=\sum f^{j} \mathrm{~d} g^{j}$ (finite sum with $f^{j}, g^{j} \in \mathcal{F}$ ) and the $\mathcal{F}$-module $\mathcal{T}(=\mathcal{T}(\mathbf{M})$ ) of vector fields $Z$ on the space $\mathbf{M}$ make a good sense. It should be noted that the vector fields are regarded as $\mathcal{F}$-linear functions $Z: \Phi \rightarrow \mathcal{F}$ where

$$
\mathrm{d} f(Z)=Z f, \varphi(Z)=Z\rfloor \varphi=\sum f^{j} Z g^{j}
$$

and if $\varphi^{1}, \varphi^{2}, \ldots$ is a basis of module $\Phi$, we denote

$$
Z=\sum z^{j} \frac{\partial}{\partial \varphi^{j}} \quad\left(\text { infinite series, arbitrary } z^{j}=\varphi^{j}(Z) \in \mathcal{F}\right)
$$

with the common abbreviation $\partial / \partial f=\partial / \partial \mathrm{d} f$. The familiar rules like

$$
\left.\mathcal{L}_{Z} f=Z f, \mathcal{L}_{Z} \varphi=Z\right\rfloor d \varphi+d \varphi(Z), \mathcal{L}_{Z} X=[Z, X], \mathcal{L}_{[X, Y]}=\mathcal{L}_{X} \mathcal{L}_{Y}-\mathcal{L}_{Y} \mathcal{L}_{X}
$$

for the Lie derivative and the Lie bracket do not need any comment.

Let $\mathbf{n}: \mathbf{N} \rightarrow \mathbf{M}$ be a mapping of manifolds. If an appropriate part of the family of functions $\mathbf{n}^{*} h^{1}, \mathbf{n}^{*} h^{2}, \ldots$ can be taken for the coordinates on $\mathbf{N}$, then $\mathbf{n}$ is called an inclusion of the submanifold $\mathbf{N}$ into the space $\mathbf{M}$. (Since $\mathbf{n}$ is injective, we occasionally identify $\mathbf{N}=\mathbf{n N} \subset \mathbf{M}$ with the subset of $\mathbf{M}$.) Analogously $\mathbf{n}$ is called a projection of $\mathbf{N}$ on the factorspace $\mathbf{M}$ if the family $\mathbf{n}^{*} h^{1}, \mathbf{n}^{*} h^{2}, \ldots$ can be completed by some additional functions to the coordinates on $\mathbf{N}$. (Since $\mathbf{n}^{*}$ is injective, we occasionally abbreviate $f=\mathbf{n}^{*} f, \varphi=\mathbf{n}^{*} \varphi$.) In order to delete singularities, we tacitly suppose the existence of $\mathcal{F}$-bases in all $\mathcal{F}$-modules to appear (unless otherwise stated). We moreover admit the localization at the points $\mathbf{P} \in \mathbf{M}$ in the sense that the $\mathcal{F}$-basis of $\mathcal{F}$-module turns into the $\mathbb{R}$-basis of $\mathbb{R}$-module after the evaluation $\mathbf{P}$ [8]. Alternatively: certain algebraical arrangements at a given point $\mathbf{P} \in \mathbf{M}$ are tacitly applied in a neighbourhood of $\mathbf{P}$ as well and this is possible on open subsets of "generic points" of $\mathbf{M}$.

\section{(II) On the diffieties [5],[8]}

For every submodule $\Omega \subset \Phi$, let $\mathcal{H}(\Omega) \subset \mathcal{T}$ be the submodule of all vector fields $Z$ such that $\Omega(Z)=0$. A submodule $\Omega \subset \Phi$ is called flat (or: satisfying the Frobenius condition) if any of the equivalent requirements

$$
\mathrm{d} \Omega \cong 0(\bmod \Omega), \mathcal{L}_{\mathcal{H}} \Omega \subset \Omega,[\mathcal{H}, \mathcal{H}] \subset \mathcal{H} \quad(\mathcal{H}=\mathcal{H}(\Omega))
$$

is satisfied. The finite-dimensional flat submodules $\Omega \subset \Phi$ are simple: the Frobenius theorem can be applied and they admit a basis consisting of total differentials. We are however interested just in the infinite-dimensional case.

Definition 2.1. A finite-codimensional submodule $\Omega \subset \Phi$ is called a diffiety if there exists a good filtration $\Omega_{*}: \Omega_{0} \subset \Omega_{1} \subset \cdots \subset \Omega=\cup \Omega_{l}$ with the finite-dimensional submodules $\Omega_{l} \subset \Omega(l=0,1, \ldots)$ such that

$$
\mathcal{L}_{\mathcal{H}} \Omega_{l} \subset \Omega_{l+1} \quad(\text { all } l), \quad \Omega_{l}+\mathcal{L}_{\mathcal{H}} \Omega_{l}=\Omega_{l+1} \quad(l \text { large enough }) .
$$

A pre-diffiety need not satisfy the codimensionality requirement.

We deal only with the diffieties unless otherwise stated. The pre-diffieties will appear later on and will be reduced to the common diffieties. 
Definition 2.2. Denoting $n(=n(\Omega))=\operatorname{dim} \Phi / \Omega=\operatorname{dim} \mathcal{H}$, functions $x_{1}, \ldots, x_{n}$ are called independent variables of diffiety $\Omega$ if the differentials $\mathrm{d} x_{1}, \ldots, \mathrm{d} x_{n}$ are linearly independent mod $\Omega$. Then the total derivatives $D_{1}, \ldots, D_{n} \in \mathcal{H}$ defined by

$$
D_{i} x_{i}=1, D_{i^{\prime}} x_{i}=0, \Omega\left(D_{i}\right)=0 \quad\left(i, i^{\prime}=1, \ldots, n ; i \neq i^{\prime}\right)
$$

constitute a basis of module $\mathcal{H}$ and the contact forms

$$
\omega\{f\}=\mathrm{d} f-\sum D_{i} f \mathrm{~d} x_{i} \quad(f \in \mathcal{F})
$$

generate the diffiety $\Omega$.

The link to the classical approach can be succinctly described as follows.

Let us consider differential equations $\mathbf{i}: \mathbf{M} \subset \mathbf{M}(m, n)$ in the sense (9). It was already clarified in Preface 1.9 that the restriction $\Omega=\mathbf{i}^{*} \Omega(m, n)$ of the module $\Omega(m, n)$ to the space $\mathbf{M}$ is a diffiety with the terms $\Omega_{l}=\mathbf{i}^{*} \Omega(m, n)_{l}$ of the good filtration. Let us conversely start with a diffiety $\Omega$. Due to (14), there exist generators

$$
\mathcal{L}_{D_{i_{1}}} \cdots \mathcal{L}_{D_{i_{r}}} \omega^{k} \quad\left(k=1, \ldots, K ; i_{1}, \ldots, i_{r}=1, \ldots, n ; r=0,1, \ldots\right)
$$

of module $\Omega$. Denoting $\omega^{k}=\sum a_{j}^{k} \mathrm{~d} h^{j}$, the Pfaffian system $\omega=0(\omega \in \Omega)$ clearly reads

$$
\sum f_{j}^{k} \frac{\partial h^{j}}{\partial x_{i}}=0, \quad D_{i_{1}} \sum f_{j}^{k} \frac{\partial h^{j}}{\partial x_{i}}=0, \quad D_{i_{1}} D_{i_{2}} \sum f_{j}^{k} \frac{\partial h^{j}}{\partial x_{i}}=0, \ldots
$$

which is a classical (infinitely prolonged) system of differential equations for a finite number of functions $h^{j}=h^{j}\left(x_{1}, \ldots, x_{n}\right)$ occuring in the forms $\omega^{1}, \ldots, \omega^{K}$.

We conclude. A diffiety supplied with a fixed choice of the dependent and the independent variables is the same as an infinitely prolonged system of differential equations.

(III) On the commutative algebra $[5],[7],[8],[9],[10]$

Conditions (14) simplify if the original filtration $\Omega_{*}$ is replaced with the gradation

$$
\operatorname{Grad} \Omega_{*}=\mathcal{M}=\mathcal{M}_{0} \oplus \mathcal{M}_{1} \oplus \cdots \quad\left(\mathcal{M}_{l}=\Omega_{l} / \Omega_{l-1}, \Omega_{-1}=0\right) .
$$

Then the Lie derivative

$$
\mathcal{L}_{D}: \Omega_{l} \rightarrow \Omega_{l+1} \quad(D \in \mathcal{H}(\Omega))
$$

turns into the $\mathcal{F}$-linear mapping $D: \mathcal{M} \rightarrow \mathcal{M}$ such that

$$
D[\omega]=\left[\mathcal{L}_{D} \omega\right] \in \mathcal{M}_{l+1} \quad\left(\omega \in \Omega_{l},[\omega] \in \mathcal{M}_{l}, D \in \mathcal{H}\right)
$$

where the square brackets denote the factorization. But more is true. Let

$$
\mathcal{A}(=\mathcal{A}(\mathcal{H}))=\mathcal{A}_{0} \oplus \mathcal{A}_{1} \oplus \cdots \quad\left(\mathcal{A}_{0}=\mathcal{F}, A_{1}=\mathcal{H}, \mathcal{A}_{2}=\mathcal{H} \odot \mathcal{H}, \ldots\right)
$$

be the algebra of homogeneous polynomials over $\mathcal{H}$. We obtain even the $\mathcal{A}$-module structure on $\mathcal{M}$. For instance

$$
\begin{gathered}
D_{i_{1}} \cdots D_{i_{r}}[\omega]=\left[\mathcal{L}_{D_{i_{1}}} \cdots \mathcal{L}_{D_{i_{r}}} \omega\right] \in \mathcal{M}_{l+r} \quad\left(\omega \in \Omega_{r}, D_{i_{1}} \cdots D_{i_{r}} \in \mathcal{A}_{r}\right), \\
D_{i_{1}} \cdots D_{i_{r}}[\omega\{f\}]=\left[\omega\left\{D_{i_{1}} \cdots D_{i_{r}} f\right\}\right] .
\end{gathered}
$$

Warning. The algebraical calculations with the $\mathcal{F}$-module are performed at a fixed point of $\mathbf{M}$. So we deal with "smooth families" of $\mathbb{R}$-modules. It follows that the classical algebra can be applied. 
In particular we recall the Hilbert polynomial

$$
\operatorname{dim} \mathcal{M}_{l}=e_{\nu}\left(\begin{array}{l}
l \\
\nu
\end{array}\right)+\cdots+e_{0}\left(\begin{array}{l}
l \\
0
\end{array}\right) \quad\left(l \text { large enough, } e_{\nu} \neq 0\right)
$$

with integer coefficients, alternatively

$$
\operatorname{dim} \Omega_{l}=e_{\nu}\left(\begin{array}{c}
l+1 \\
\nu+1
\end{array}\right)+\cdots+e_{-1}\left(\begin{array}{c}
l+1 \\
0
\end{array}\right) \quad\left(l \text { large enough, } e_{\nu} \neq 0\right) .
$$

Then the degree $\nu(=\nu(\Omega))$ and the integer $\mu(=\mu(\Omega))=e_{\nu}>0$ do not depend on the choice of the filtration $\Omega_{*}$.

Definition 2.3. We claim that the solution of diffiety $\Omega$ depends on $\mu(\Omega)$ functions of $\nu(\Omega)+1$ variables. Diffiety $\Omega$ is overdetermined, determined, or underdetermined according to whether $\nu+1<n-1, \nu+1=$ $n-1$, or $\nu+1>n-1$, respectively.

One can observe that we have intentionally not yet introduced the concept of a solution of diffiety $\Omega$. Definition 2.3 therefore designates a more formal property of diffiety $\Omega$, however, it is in full accordance with quite opposite approach [11],[12],[13],[14],[15],[16] in the theory of exterior differential systems.

Remark. The above reasonings make sense even for the much easier case of the finite-dimensional underlying space $\mathbf{M}$ which need not be separately discussed here. Let us only recall the familiar Frobenius theorem which ensures that then the diffiety $\Omega \subset \Phi(\mathbf{M})$ has a basis $\mathrm{d} f^{1}, \ldots, \mathrm{d} f^{\mu}$ and the solution depends on $\mu=\operatorname{dim} \Omega=e_{-1}$ parameters. We formally put $\nu(=\nu(\Omega))=-1$.

\section{(IV) On the symmetries $[5],[7]$}

Admissible mappings $\mathbf{m}: \mathbf{M} \rightarrow \overline{\mathbf{M}}$ between manifolds satisfy $\mathbf{m}^{*} \mathcal{F}(\overline{\mathbf{M}}) \subset \mathcal{F}(\mathbf{M})$ whence $\mathbf{m}^{*} \Phi(\overline{\mathbf{M}}) \subset$ $\Phi(\mathbf{M})$.

Definition 2.4. Let $\Omega \subset \Phi(\mathbf{M})$ and $\bar{\Omega} \subset \Phi(\overline{\mathbf{M}})$ be diffieties. Then the mapping $\mathbf{m}: \mathbf{M} \rightarrow \overline{\mathbf{M}}$ is said to be a morphism of diffieties if $\mathbf{m}^{*} \bar{\Omega} \subset \Omega$. Invertible morphisms with $n(\Omega)=\bar{n}(\Omega)$ are isomorphisms and then $\mathbf{m}^{*} \bar{\Omega}=\Omega$. Assuming $\mathbf{M}=\overline{\mathbf{M}}$ and $\Omega=\bar{\Omega}$, invertible morphisms are called symmetries (or: automorphisms).

Three subcases of symmetries can be informally mentioned as follows. First, if a given filtration is preserved (Figure 1a). Second, if an unknown filtration is preserved (Figure 1b). Third, if no finitedimensional subspace of $\Omega$ is preserved (Figure 1c).

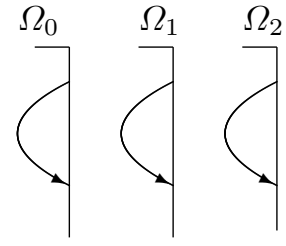

(1a)

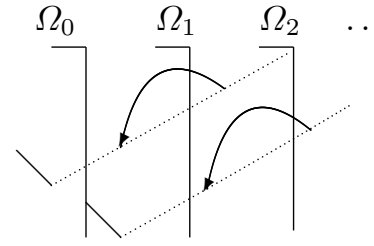

(1b)

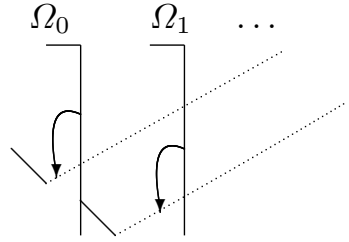

(1c)

Figure 1: Subcases of symmetries.

The common methods of the general equivalence [16] can be directly applied only to the subcase (1a) and with slight adaptations even to the subcase (1b) not occuring in the classical theory. Alas, the common methods fail in the subcase (1c). No universal finite algorithm for the determination of all symmetries or equivalences of diffieties is known. 
(V) On the variations [5],[7]

We expect that the determination of "approximative symmetries" is easier. They are realized by vector fields.

Definition 2.5. Variations $Z \in \mathcal{T}(\mathbf{M})$ of a diffiety $\Omega \subset \Phi(\mathbf{M})$ are defined by the condition $\mathcal{L}_{Z} \Omega \subset \Omega$. Variations generating a Lie group are called infinitesimal symmetries.

The Figure 1 with arrows denoting the Lie derivative $\mathcal{L}_{Z}$ can be mentioned as well, however, the comments are different compared to the previous case of the symmetries $\mathbf{m}$. In more detail, the subcases (1a) and (1b) concern the variations $Z$ which generate a group, that is, we have the infinitesimal symmetries. Then, paradoxically, the "true variations" of the subcase (1c) do not cause much difficulties.

Lemma 2.1. A variation $Z$ is characterized by the property

$$
\left(\mathcal{L}_{D} \omega\right)(Z)=D \omega(Z) \quad(D \in \mathcal{H}, \omega \in \Omega) .
$$

Proof. Inclusion $\mathcal{L}_{Z} \Omega \subset \Omega$ is equivalent to the identity $\mathcal{L}_{Z} \mathcal{H}=[Z, \mathcal{H}] \subset \mathcal{H}$ which follows from the equations

$$
0=\Omega(\mathcal{H}), 0=Z(\Omega(\mathcal{H}))=\mathcal{L}_{Z} \Omega(\mathcal{H})+\Omega([Z, \mathcal{H}]) .
$$

So we have

$$
D(\omega(Z))=\mathcal{L}_{D} \omega(Z)+\omega([D, Z])=\mathcal{L}_{D} \omega(Z) .
$$

This simple Lemma 2.1 involves the common linearization procedure [12],[13] as a particular subcase when $\omega=\omega\{f\}$. If a variation $Z$ is represented by the series (13) then the coefficients $\varphi^{j}(Z)$ with appropriately chosen forms $\varphi^{j} \in \Omega$ can be effectively described. On the contrary, the study of the infinitesimal symmetries $Z$ is rather difficult: they satisfy one additional condition, the invariance of some filtration. In general, there are too many filtrations and this prevents us from resolving the symmetry problem completely.

\section{(VI) On the evolutional diffieties}

The infinitesimal symmetry $Z \in \mathcal{T}(\mathbf{M})$ of a diffiety $\Omega \subset \Phi(\mathbf{M})$ is a classical concept with simple geometrical significance, the flow on the underlying space $\mathbf{M}$. The variations $Z$ look rather ambiguous in this respect, they are rather arguably identified with virtual flows on the vague space of solutions of diffiety $\Omega$ [12],[13]. A rigorous view is however possible [5].

Let us introduce the direct product $\overline{\mathbf{M}}=\mathbf{M} \times \mathbb{R}$ of manifolds with coordinate $t$ in the factor $\mathbb{R}$. Omitting the technicalities, a function $f \in \Phi(\mathbf{M})$ can be regarded as a function on $\overline{\mathbf{M}}$ (independent of $t$ ) and analogously for the forms $\varphi \in \Phi(\mathbf{M})$. If $\varphi^{1}, \varphi^{2}, \ldots$ is a basis of module $\Phi(\mathbf{M})$ then $d t, \varphi^{1}, \varphi^{2}, \ldots$ constitute a basis of $\Phi(\overline{\mathbf{M}})$. The vector fields $\bar{Z} \in \mathcal{T}(\overline{\mathbf{M}})$ can be described as follows. There are horizontal vector fields $H \in \mathcal{T}(\overline{\mathbf{M}})$ satisfying $H t=0$ and they may be identified with vector fields $H(t) \in \mathcal{T}(\mathbf{M})$ depending on parameter $t$ which are distributed over $\overline{\mathbf{M}}$ by the $t$-shifts along the $\mathbb{R}$-component. Then, by using the obvious vertical vector field $\partial / \partial t$, we obtain the unique decomposition

$$
\bar{Z}=H+\bar{f} \frac{\partial}{\partial t} \quad(\bar{f}=\bar{Z} t, H t=0, H f=H(t) f \text { if } f \in \mathcal{F}(\mathbf{M}) \subset \mathcal{F}(\overline{\mathbf{M}}))
$$

into the horizontal and the vertical summands.

With this preparation, the following diffieties of rather special kind provide the rigorous geometrical sense of the variations.

Definition 2.6. Let $Z(t) \in \mathcal{T}(\mathbf{M})$ be a variation depending on parameter $t$ of a diffiety $\Omega \subset \Phi(\mathbf{M})$. We introduce the evolutional diffiety $\bar{\Omega} \subset \Phi(\mathbf{M} \times \mathbb{R})$ with generators

$$
\omega(Z(t)) d t-\omega \in \bar{\Omega} \subset \Phi(\overline{\mathbf{M}}) \quad(\omega \in \Omega) .
$$


Alternatively, the module $\mathcal{H}(\bar{\Omega})$ is generated by all vector fields

$$
E=Z(t)+\frac{\partial}{\partial t} \in \mathcal{H}(\bar{\Omega}) \subset \mathcal{T}(\overline{\mathbf{M}}) \text { and } D \in \mathcal{H}(\Omega)
$$

where the vector fields $D \in \mathcal{H}(\Omega)$ are identified with horizontal vector fields distributed over $\mathbf{M} \times \mathbb{R}$.

Definition 2.7. An inclusion $\mathbf{n}: \mathbf{N} \rightarrow \mathbf{M}\left(\mathbf{N} \subset \mathbb{R}^{n}, n=n(\Omega)\right)$ is said to be a solution of diffiety $\Omega \subset \Phi(\mathbf{M})$ if $\mathbf{n}^{*} \Omega=0$.

In accordance with tradition, we identify $\mathbf{N}=\mathbf{n N}$ which better corresponds to the intuition: the diffiety $\Omega$ identically vanishes on the subspace $\mathbf{N}=\mathbf{n} \mathbf{N} \subset \mathbf{M}$ of the total space or, equivalently, all vector fields $D \in \mathcal{H}(\Omega)$ are tangent to this subspace $\mathbf{N}=\mathbf{n N} \subset \mathbf{M}$ of the dimension $n=n(\Omega)$. We recall that the existence of solutions is a highly delicate task [11],[17],[15], to put it mildly. (Informally, this is in fact a mere rigid formalism for the familiar concept: if $x_{1}, \ldots, x_{n}$ are independent variables of diffiety $\Omega \subset \Phi(\mathbf{M})$ and $h^{1}, h^{2}, \ldots$ are coordinates on $\mathbf{M}$ then the solution is given by such formulae $h^{j}=h^{j}\left(x_{1}, \ldots, x_{n}\right)$ that satisfy the Pfaffian system $\omega=0(\omega \in \Omega)$.)

The resulting point is as follows. Let $\overline{\mathbf{N}} \subset \overline{\mathbf{M}}$ be a solution of the evolutional diffiety $\bar{\Omega}$, hence $\operatorname{dim} \overline{\mathbf{N}}=\operatorname{dim} \mathcal{H}(\bar{\Omega})$ and vector fields $\bar{D} \in \mathcal{H}(\bar{\Omega})$ are tangent to $\overline{\mathbf{N}}$. Let $\mathbf{N} \subset \mathbf{M}$ be the projection of $\overline{\mathbf{N}}$. Since the vector fields $D$ distributed over $\overline{\mathbf{M}}$ are tangent to $\overline{\mathbf{N}}$, we conclude that the projections of the level subsets $t=$ const. of $\overline{\mathbf{N}}$ on $\mathbf{N}$ are solutions of $\Omega$. On the other hand, $E$ is tangent to $\overline{\mathbf{N}}$ as well and generates a one-parameter group on $\overline{\mathbf{N}}$ where the $\mathbb{R}$-component involves mere translations $t \rightarrow t+c$. It follows that the level sets on $\overline{\mathbf{N}}$ are permuted and the projections of the level sets in $\mathbf{N}$, the solutions of $\Omega$, are permuted as well.

We summarize: a variation (possibly depending on a parameter) generates many flows, but each only on a rather narow family of solutions of diffiety $\Omega$.

Proposition 2.1. Let $\overline{\mathbf{N}} \subset \overline{\mathbf{M}}=\mathbf{M} \times \mathbb{R}$ be a solution of the evolutional diffiety $\bar{\Omega} \subset \Phi(\overline{\mathbf{M}})$ and $\mathbf{N} \subset \mathbf{M}$ the natural projection of $\overline{\mathbf{N}}$. Then the level subsets $t=$ const. of $\overline{\mathbf{N}}$ are projected on the solutions of diffiety $\Omega \subset \Phi(\mathbf{M})$. The vector field $E \in \mathcal{T}(\overline{\mathbf{M}})$ generates a Lie group on $\overline{\mathbf{N}}$ and its projection $Z(t) \in \mathcal{T}(\mathbf{M})$ permutes the solutions of $\Omega$ contained in $\mathbf{N}$.

The multi-parameter evolution diffieties for the case of a finite-dimensional Lie algebra of variations $Z$ can be analogously introduced as well.

Remark. A huge literature on the infinitesimal symmetries in finite-order jet spaces exists. These are the order-preserving Lie symmetries of Figure 1a. We cannot refer to any literature systematically devoted to the order-destroying symmetries of Figure 1b. The main and rather original ideas of monograph [13] concern the abstract theory of variations of Figure 1c, alas, with lack of any convincing examples and applications. The alternative approach [12],[13] to the diffieties is worth mentioning, however, large preparatory parts are devoted to finite-order jet spaces and neither the results nor the methods of proofs can be carried over the diffieties, they are even misleading in many respects.

\section{The Involutiveness}

In the classical external theory, the involutivity ensures that a given finite system of differential equations is compatible. In the classical internal theory, the involutivity ensures the same for a finite Pfaffian system. We are interested in diffieties $\Omega$ where the compatibility is already attained. Then the involutivity describes the structure of the higher-order summands of the $\mathcal{A}$-module $\mathcal{M}$ corresponding to a given good filtration $\Omega_{*}$ and this is a mere algebra.

Let $Z_{1}, \ldots, Z_{n}(n=n(\Omega))$ be a basis of module $\mathcal{H}(=\mathcal{H}(\Omega))$ and $\mathcal{A}(i) \subset \mathcal{A}(i=0, \ldots, n)$ the ideal generated by $Z_{1}, \ldots, Z_{i}$. In particular $\mathcal{A}(0)=0$ and

$$
\mathcal{A}(n)=\mathcal{A}_{1} \oplus \mathcal{A}_{2} \oplus \cdots=\mathfrak{m} \subset \mathcal{A} \quad\left(\mathcal{A}_{1}=\mathcal{H}, \mathcal{A}_{2}=\mathcal{H} \odot \mathcal{H}, \ldots\right)
$$


is the maximal ideal. We introduce the factormodules

$$
\mathcal{M}(i)=\mathcal{M} / \mathcal{A}(i) \mathcal{M}=\mathcal{M}(i)_{0} \oplus \mathcal{M}(i)_{1} \oplus \cdots \quad\left(\mathcal{M}(i)_{l}=\mathcal{M}_{l} / \mathcal{A}(i) \mathcal{M} \cap \mathcal{M}_{l}\right)
$$

which are $\mathcal{A}$-modules as well. In particular

$$
Z_{i+1}: \mathcal{M}(i)_{l} \rightarrow \mathcal{M}(i)_{l+1} \quad(i=0, \ldots, n-1) .
$$

Definition 3.1. The basis $Z_{1}, \ldots, Z_{n}$ is called ordinary (or: generic [5]) for a given good filtration $\Omega_{*}$ if (18) are injections for $l$ large enough.

Theorem 3.1 ([5],[8]). There exists an ordinary basis of $\mathcal{H}$.

In full generality, this is rather nontrivial result, however, for all current examples, such a basis can be easily found: the vector fields $Z_{1}, Z_{2}, \ldots$ should not be too special, see also below. We are passing to the main topic.

Definition 3.2. The basis $Z_{1}, \ldots, Z_{n}$ of $\mathcal{H}$ is called quasiregular if (18) are injections for all $l \geq 1$. A filtration $\Omega_{*}$ is called involutive if there exists a quasiregular basis and moreover $\mathcal{H M}_{l}=\mathcal{M}_{l+1}(l \geq 0)$.

The last condition is clearly equivalent to $\Omega_{l}+\mathcal{L}_{\mathcal{H}} \Omega_{l}=\Omega_{l+1}(l \geq 0)$ which can be ensured by a simple change of the original filtration. Let us introduce the $c$-lift $\Omega_{*+c}(c=0,1, \ldots)$ of the original filtration $\Omega_{*}$ such that

$$
\Omega_{*+c}=\bar{\Omega}_{*}: \bar{\Omega}_{0}=\Omega_{c} \subset \bar{\Omega}_{1}=\Omega_{c+1} \subset \cdots \subset \Omega=\cup \bar{\Omega}_{l}=\cup \Omega_{l+c} .
$$

Due to the second requirement (13), it follows easily that Theorem 3.1 is equivalent to:

Theorem 3.2. Every lift $\Omega_{*+c}$ with c large enough is involutive.

This provides the essence of all prolongations into the involutivity mechanisms [11],[16],[5],[18]. Also the singular solutions can be included, see below.

We conclude with a brief description of the ordinary basis. The first term $Z_{1}$ appears as follows [8]. There is a finite family of prime ideals $\mathfrak{p} \subset \mathcal{A}, \mathfrak{p} \neq \mathcal{A}$, associated to the module $\mathcal{M}$, that is, such that there exists a submodule of $\mathcal{M}$ isomorphic to $\mathcal{A} / \mathfrak{p}$. The multiplications $Z: \mathcal{M}_{l} \rightarrow \mathcal{M}_{l+1}(Z \in \mathcal{H})$ are all injective if and only if

$$
Z \notin \mathcal{A}_{1} \cap(\cup \mathfrak{p})=\mathcal{H} \cap(\cup \mathfrak{p}) .
$$

If all $\mathfrak{p} \neq \mathfrak{m}$ then such $Z=Z_{1}$ does exist. If however $\mathfrak{m}$ belongs to the associated ideals then the submodules of $\mathcal{M}$ isomorphic to $\mathcal{A} / \mathfrak{m}=\mathcal{F}$ can be included into the submodule $\mathcal{M}_{0} \subset \mathcal{M}$ if the original filtration $\Omega_{*}$ is replaced with a $c$-lift large enough. The injectivity $Z=Z_{1}: \mathcal{M}_{l} \rightarrow \mathcal{M}_{l+1}(l \geq 1)$ is ensured. The following terms $Z_{2}, \ldots, Z_{n}$ appear by analogous construction applied to the $\mathcal{A}$-modules $\mathcal{M}(1), \ldots, \mathcal{M}(n-1)$ instead of $\mathcal{M}=\mathcal{M}(0)$.

Altogether we conclude that the terms $Z_{1}, \ldots, Z_{n}$ of the ordinary basis should not lie in a finite family of certain linear subspaces of the $\mathcal{F}$-module $\mathcal{H}$. We will succinctly express this property by saying that they are "not too special". It follows that the total derivatives $D_{1}, \ldots, D_{n} \in \mathcal{H}$ are "not too special" for an appropriate "not too special" choice of the independent variables $x_{1}, \ldots, x_{n}$.

Remark. The primary approach to the compatibility of the systems of differential equations directly use the commutativity $\partial^{2} / \partial x \partial y=\partial^{2} / \partial y \partial x$ of various second derivatives which results in perfect ultimate theory [14]. However, though this theory can be effectively applied to particular problems, the calculations strongly depend on subtle formal details. On the contrary, the É. Cartan's involutivity [15] subsequently completed with the prolongation procedure [16] is of the geometrical nature. In the actual rigorous expositions, this topic belongs to the most difficult tasks even though the powerful tools of the commutative and the homological algebra are applied [11],[18]. The classical involutivity concept differs from ours in Definition 3.2, since the involutivity of a finite--order system of differential equations and of a finite Pfaffian system is introduced [11],[17],[15],[16],[5] and Theorem 3.2 declares the involutivity of every Pfaffian system $\omega=0\left(\omega \in \Omega_{l}, l\right.$ fixed and large enough) in the common classical sense, that 
is, we have involutivity together with the prolongation procedure involved. It should be noted that the involutivity ensures the finite length of algorithms. This concerns the case (1a) of Figure 1 and prevents us from completely discussing the remaining cases (1b) and (1c) in the present time.

We recall that except for some advanced applications in geometry [11],[17],[16], the involutivity can be easily verified in all current examples of the analysis. On the other hand, the following technical tool of standard filtrations directly inspired just by the abstract involutivity seems to be useful even for the solution of various "simple" earthly problems to follow.

\section{The Standard Filtrations}

Theorems 3.1 and 3.2 concern the higher-order terms $\mathcal{M}[i]_{l}$ of the $\mathcal{A}$-module $\mathcal{M}[i]$. Returning to the original filtration $\Omega_{*}$, they describe a certain property of the higher-order terms $\Omega_{l}$ of a good filtration $\Omega_{*}$.

For given vector fields $Z_{1}, Z_{2}, \ldots \in \mathcal{H}$, let us introduce the large series of accompanying "rough" filtrations

$$
\begin{aligned}
& \Omega\left(Z_{1}\right)_{*}: \Omega\left(Z_{1}\right)_{0} \subset \Omega\left(Z_{1}\right)_{1} \subset \ldots \subset \Omega \quad\left(\Omega\left(Z_{1}\right)_{l}=\sum \mathcal{L}_{Z_{1}}^{k} \Omega_{l}\right), \\
& \Omega\left(Z_{1}, Z_{2}\right)_{*}: \Omega\left(Z_{1}, Z_{2}\right)_{0} \subset \Omega\left(Z_{1}, Z_{2}\right)_{1} \subset \ldots \subset \Omega\left(\Omega\left(Z_{1}, Z_{2}\right)_{l}=\sum \mathcal{L}_{Z_{2}}^{k} \Omega\left(Z_{1}\right)_{l}\right), \\
& \ldots
\end{aligned}
$$

of diffiety $\Omega$. For every submodule $\Theta \subset \Phi$ and a vector field $Z \in \mathcal{H}(\Theta)$ we moreover introduce the submodule $\operatorname{Ker}_{Z} \Theta \subset \Theta$ of all $\vartheta \in \Theta$ with $\mathcal{L}_{Z} \vartheta \in \Theta$. (The latter concept will be applied only in the particular case when $\Theta \subset \Omega$ and $Z \in \mathcal{H}=\mathcal{H}(\Omega) \subset \mathcal{H}(\Theta)$.) One can see that Theorems 3.1 and 3.2 are equivalent to the equalities

$$
\begin{aligned}
\operatorname{Ker}_{Z_{1}} \Omega_{l+1} & =\Omega_{l}, \\
\operatorname{Ker}_{Z_{2}} \Omega\left(Z_{1}\right)_{l+1} & =\Omega\left(Z_{1}\right)_{l}, \\
\operatorname{Ker}_{Z_{3}} \Omega\left(Z_{1}, Z_{2}\right)_{l+1} & =\Omega\left(Z_{1}, Z_{2}\right)_{l}, \\
\ldots &
\end{aligned}
$$

valid for $l$ large enough and not too special $Z_{1}, Z_{2}, \ldots \in \mathcal{H}$. Our next aim is to ensure (20) for all values of $l$ after appropriate adjustements and this is possible if certain obstructions $\mathcal{R}^{0}, \mathcal{R}^{1}, \ldots$ are absent.

Let us start with the first equality (20). Abbreviating $X=Z_{1}$, we may suppose $\operatorname{Ker}_{X} \Omega_{l}=\Omega_{l-1}(l \geq L)$ and consider the inclusions

$$
\cdots \supset \Omega_{L}=\operatorname{Ker}_{X} \Omega_{L+1} \supset \Omega_{L-1}=\operatorname{Ker}_{X} \Omega_{L} \supset \operatorname{Ker}_{X}^{2} \Omega_{L} \supset \operatorname{Ker}_{X}^{3} \Omega_{L} \supset \cdots
$$

The strict inclusions terminate with certain equalities $\operatorname{Ker}_{X}^{k} \Omega_{L}=\operatorname{Ker}_{X}^{k+1} \Omega_{L}(k \geq K)$ and so we obtain the new filtration

$$
\begin{aligned}
& \bar{\Omega}_{*}: \bar{\Omega}_{0}=\operatorname{Ker}_{X}^{K-1} \Omega_{L} \subset \bar{\Omega}_{1}=\operatorname{Ker}_{X}^{K-2} \Omega_{L} \subset \ldots \\
& \subset \bar{\Omega}_{K-2}=\operatorname{Ker}_{X} \Omega_{L} \subset \bar{\Omega}_{K-1}=\Omega_{L} \subset \bar{\Omega}_{K}=\Omega_{L+1} \subset \ldots
\end{aligned}
$$

of diffiety $\Omega$ with strict inclusions together with the submodule

$$
\mathcal{R}^{0}=\operatorname{Ker}_{X}^{K} \Omega_{L}=\operatorname{Ker}_{X} \bar{\Omega}_{0} \subset \bar{\Omega}_{0} \subset \Omega .
$$

Theorem 4.1 ([5],[8],[9]). Filtration (21) does not depend on the choice of $X$. The module $\mathcal{R}^{0}$ is flat and does not depend even on the choice of the original good filtration $\Omega_{*}$.

So we may denote $\mathcal{R}^{0}=\mathcal{R}^{0}(\Omega)$ and there are equalities

$$
\operatorname{Ker}_{X} \bar{\Omega}_{l+1}=\bar{\Omega}_{l}(l>0), \operatorname{Ker}_{X} \bar{\Omega}_{0}=\mathcal{R}^{0}, \operatorname{Ker}_{X} \mathcal{R}^{0}=\mathcal{R}^{0}
$$

corresponding to the injections

$$
X: \overline{\mathcal{M}}_{l} \rightarrow \overline{\mathcal{M}}_{l+1}(l>0), \overline{\mathcal{M}}_{0} / \mathcal{R}^{0} \rightarrow \overline{\mathcal{M}}_{1}\left(\overline{\mathcal{M}}_{l}=\bar{\Omega}_{l} / \bar{\Omega}_{l-1}\right)
$$

for all not too special vector fields $X \in \mathcal{H}$. The first equality (20) is universal if and only if $\mathcal{R}^{0}=0$ is the trivial module. 
Passing to the second equality (20), the reasonings will be applied to "modulo $Z_{1}$ " as follows. We abbreviate $Y=Z_{2}$ and consider the inclusions

$$
\cdots \supset \Omega(X)_{L} \supset \Omega(X)_{L-1}=\operatorname{Ker}_{Y} \Omega(X)_{L} \supset \operatorname{Ker}_{Y}^{2} \Omega(X)_{L} \supset \operatorname{Ker}_{Y}^{3} \Omega(X)_{L} \supset \cdots
$$

with $L$ large enough. The strict inclusions again terminate and we obtain the new filtration

$$
\begin{aligned}
& \bar{\Omega}(X)_{*}: \bar{\Omega}(X)_{0}=\operatorname{Ker}_{Y}^{K-1} \Omega(X)_{L} \subset \bar{\Omega}(X)_{1}=\operatorname{Ker}_{Y}^{K-2} \Omega(X)_{L} \subset \ldots \\
& \subset \bar{\Omega}(X)_{K-2}=\operatorname{Ker}_{Y} \Omega(X)_{L} \subset \bar{\Omega}(X)_{K-1}=\Omega(X)_{L} \subset \bar{\Omega}(X)_{K}=\Omega(X)_{L+1} \subset \ldots
\end{aligned}
$$

of diffiety $\Omega$ with strict inclusions together with the module

$$
\mathcal{R}^{1}=\operatorname{Ker}_{Y}^{K} \Omega(X)_{L}=\operatorname{Ker}_{Y} \bar{\Omega}(X)_{0} \subset \bar{\Omega}(X)_{0} \subset \Omega .
$$

Theorem 4.2 ([8],[9]). Filtration (22) does not depend on the choice of $Y$. The module $\mathcal{R}^{1}$ is flat and does not depend even on the choice of the original good filtration $\Omega_{*}$.

We may denote $\mathcal{R}^{1}=\mathcal{R}^{1}(\Omega)$ and there are equalities

$$
\operatorname{Ker}_{Y} \bar{\Omega}(X)_{l+1}=\bar{\Omega}(X)_{l}(l>0), \operatorname{Ker}_{Y} \bar{\Omega}(X)_{0}=\mathcal{R}^{1}, \operatorname{Ker}_{Y} \mathcal{R}^{1}=\mathcal{R}^{1}
$$

corresponding to the injections

$$
Y: \overline{\mathcal{M}}[1]_{l} \rightarrow \overline{\mathcal{M}}[1]_{l+1}(l>0), \overline{\mathcal{M}}[1]_{0} / \mathcal{R}^{1} \rightarrow \overline{\mathcal{M}}[1]_{1}\left(\overline{\mathcal{M}}[1]_{l}=\bar{\Omega}(X)_{l} / \bar{\Omega}(X)_{l-1}\right)
$$

for all not too special vector fields $Y \in \mathcal{H}$. The second equality (20) is universal if and only if $\mathcal{R}^{1}=0$ is the trivial module.

The procedure can be continued with $Z_{3}, Z_{4}, \ldots$ as well with quite analogous result. We obtain certain filtrations

$$
\bar{\Omega}_{*}, \bar{\Omega}\left(Z_{1}\right)_{*}, \bar{\Omega}\left(Z_{1}, Z_{2}\right)_{*}, \ldots
$$

which are good in the common sense that

$$
\mathcal{L}_{\mathcal{H}} \bar{\Omega}(\cdot)_{l} \subset \bar{\Omega}(\cdot)_{l}(\text { all } l), \quad \bar{\Omega}(\cdot)_{l}+\mathcal{L}_{\mathcal{H}} \bar{\Omega}(\cdot)_{l}=\bar{\Omega}(\cdot)_{l+1}(l \text { large enough })
$$

and moreover standard in the sense that

$$
\begin{aligned}
& \operatorname{Ker}_{Z_{k+1}} \bar{\Omega}\left(Z_{1}, \ldots, Z_{k}\right)_{l+1}=\bar{\Omega}\left(Z_{1}, \ldots, Z_{k}\right)_{l} \quad(l>0) \\
& \operatorname{Ker}_{Z_{k+1}} \bar{\Omega}\left(Z_{1}, \ldots, Z_{k}\right)_{0}=\mathcal{R}^{k}, \operatorname{Ker}_{Z_{k+1}} \mathcal{R}^{k}=\mathcal{R}^{k}
\end{aligned}
$$

The procedure becomes trivial if $k>\nu=\nu(\Omega)$ since then

$$
\Omega\left(Z_{1}, \ldots, Z_{k}\right)_{l}=\Omega(l \text { large enough }), \bar{\Omega}\left(Z_{1}, \ldots, Z_{k}\right)_{l}=\Omega, \mathcal{R}^{k}=\Omega .
$$

The resulting residual submodules $\mathcal{R}^{k} \subset \Omega$ constitute the controllability series

$$
\mathcal{R}^{0} \subset \mathcal{R}^{1} \subset \cdots \subset \mathcal{R}^{\nu+1}=\Omega,
$$

to be discussed below in more detail. We speak of a controllable diffiety $\Omega$ if $\mathcal{R}^{0}=\cdots=\mathcal{R}^{\nu}=0$ are trivial modules.

\section{Ordinary Differential Equations}

We interrupt the general theory in order to clearly demonstrate the substance of the above constructions. Let us mention the relatively simple diffieties $\Omega \subset \Phi(\mathbf{M})$ with one independent variable $x=x_{1}$ (abbreviation). Let $\Omega_{*}: \Omega_{0} \subset \Omega_{1} \subset \cdots \subset \Omega$ be a good filtration. We recall the mappings

$$
\mathcal{L}_{D}: \Omega_{l} \rightarrow \Omega_{l+1}, Z: \mathcal{M}_{l} \rightarrow \mathcal{M}_{l+1} \quad\left(\mathcal{M}_{l}=\Omega_{l} / \Omega_{l-1}, Z \in \mathcal{H}\right)
$$


Since $\mathcal{H}=\mathcal{H}(\Omega) \subset \mathcal{T}(\mathbf{M})$ is one-dimensional submodule, then, omitting the trivial subcase $\operatorname{dim} \mathbf{M}<\infty$, we have the Hilbert polynomial

$$
\operatorname{dim} \mathcal{M}_{l}=c_{0}=\mu(\Omega)>0 \quad(l \text { large enough })
$$

The involutivity becomes trivial, however, the standard filtration (21) with any nonvanishing vector field $X=Z_{1} \in \mathcal{H}(\Omega)$ is worth mentioning.

We may choose $X=D=D_{1}$ the total derivative defined by the properties

$$
D x=1, \omega(D)=0 \quad(\omega \in \Omega)
$$

(In particular examples to follow, explicit formulae will be stated.) In accordance with general theory, let us suppose

$$
\operatorname{Ker}_{D} \Omega_{l+1}=\Omega_{l} \quad(l \geq L), \quad \operatorname{Ker}_{D}^{k} \Omega_{L}=\operatorname{Ker}_{D}^{k+1} \Omega_{L} \quad(k \geq K) .
$$

Then the standard filtration

$$
\bar{\Omega}_{*}: \bar{\Omega}_{0}=\operatorname{Ker}_{D}^{K-1} \Omega_{L} \subset \cdots \subset \bar{\Omega}_{K-2}=\operatorname{Ker}_{D} \Omega_{L} \subset \bar{\Omega}_{K-1}=\Omega_{L} \subset \bar{\Omega}_{K}=\Omega_{L+1} \subset \cdots
$$

together with the flat submodule

$$
\mathcal{R}^{0}=\operatorname{Ker}_{D}^{K} \Omega_{L}=\operatorname{Ker}_{D} \bar{\Omega}_{0} \subset \Omega_{0} \quad\left(\operatorname{Ker}_{D} \mathcal{R}^{0}=\mathcal{R}^{0}\right)
$$

easily appears by a merely linear algebra. (We again refer to examples for quite explicit results. Warning: the following construction of the standard basis cannot be directly generalized for the case of several independent variables.) In the meantime, we also obtain a rather useful basis of diffiety $\Omega$ as a by-product. This is made as follows. Let us choose a basis

$\tau^{r}\left(r=1, \ldots, R=\operatorname{dim} \mathcal{R}^{0}\right)$ of module $\mathcal{R}^{0}$ and then together with

$\pi_{0}^{j}\left(j=1, \ldots, j_{0}\right)$ basis of the module $\bar{\Omega}_{0}$ and then together with

$\pi_{1}^{j}=\mathcal{L}_{D} \pi_{0}^{j}(j$ as above $), \pi_{0}^{j^{\prime}}\left(j^{\prime}=j_{0}+1, \ldots, j_{1}\right)$ of module $\bar{\Omega}_{1}$, and then with

$\pi_{2}^{j}=\mathcal{L}_{D}^{2} \pi_{0}^{j}, \pi_{1}^{j^{\prime}}=\mathcal{L}_{D} \pi_{0}^{j^{\prime}}, \pi_{0}^{j^{\prime \prime}}\left(j^{\prime \prime}=j_{1}^{\prime}+1, \ldots, j_{2}^{\prime}\right)$ of module $\bar{\Omega}_{2}$,

$\cdots$.

The procedure in a certain sense stops. The identity

$$
\operatorname{Ker}_{D} \bar{\Omega}_{K}=\operatorname{Ker}_{D} \Omega_{L+1}=\Omega_{L}=\bar{\Omega}_{K-1}
$$

implies $j_{K}=j_{K+1}$ and analogously $j_{K+1}=j_{K+2}=\cdots$ as well. We obtain only a finite number $j_{k}$ of initial forms

$$
\pi_{0}^{1}, \ldots, \pi_{0}^{j_{0}} \in \bar{\Omega}_{0} ; \pi^{j_{0}+1}, \ldots, \pi_{0}^{j_{1}} \in \bar{\Omega}_{1} ; \ldots ; \pi_{0}^{j_{K-1}+1}, \ldots, \pi_{0}^{j_{K}} \in \bar{\Omega}_{K}
$$

with the lower zero indices and they provide the so called standard basis

$$
\tau^{r} \quad(r=1, \ldots, R), \quad \pi_{s}^{j}=\mathcal{L}_{D}^{s} \pi_{0}^{j} \quad\left(j=1, \ldots, j_{K} ; s=0,1, \ldots\right)
$$

of diffiety $\Omega$. In fact $j_{K}=c_{0}=\mu(\Omega)$ follows from (26) and since $\mathcal{R}^{0}$ is flat, there exists even a basis $\tau^{r}=d t^{r}\left(r=1, \ldots, R=\operatorname{dim} \mathcal{R}^{0}\right)$.

The result can be transparently visualized (see Figure 2 ): the original "cross-arrows $\mathcal{L}_{Z}$ " are "collected" in $\mathcal{R}^{0}$ and only the infinite sequences $\pi_{r}^{j}=\mathcal{L}_{D}^{r} \pi_{0}^{j}(j=1, \ldots, \mu(\Omega) ; r=0,1, \ldots)$ without any crossing remain. 


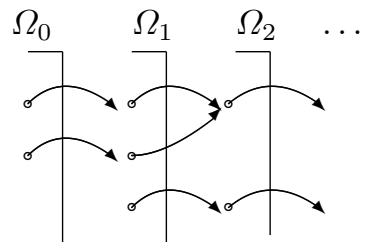

(2a) the original filtration

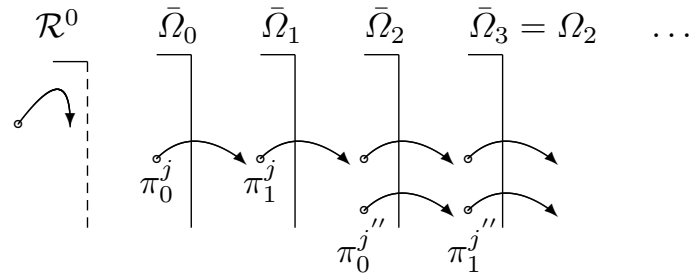

(2b) the standard filtration

Figure 2: Filtrations.

Some immediate consequences of this construction both for the general theory and for the practice of calculations are as follows.

\subsection{The Uniqueness}

We recall that the module $\mathcal{R}^{0}$ is unique. The standard filtration $(27)$ is unique if $\mu(\Omega)=1$ and we refer to quite simple proof $[7$, Theorem 26]. This is the main result of the stimulating article [16] where the concept of the absolute theory was introduced for the first time. Close connection to the beautiful explicit solvability Monge problem [19],[20],[5] is worth mentioning, see also below.

\subsection{The Morphisms [7]}

If $\mathbf{m}: \mathbf{M} \rightarrow \mathbf{M}$ is a morphism of $\Omega$ then

$$
D \mathbf{m}^{*} x \cdot \mathbf{m}^{*} \mathcal{L}_{D} \omega=\mathcal{L}_{D} \mathbf{m}^{*} \omega \quad(\omega \in \Omega)
$$

and in terms of the standard basis

$$
\mathbf{m}^{*} \tau^{r} \in \mathcal{R}^{0}, D \mathbf{m}^{*} x \cdot \mathbf{m}^{*} \pi_{s+1}^{j}=\mathcal{L}_{D} \mathbf{m}^{*} \pi_{s}^{j} \quad(\text { all } r, j, s) .
$$

Such a morphism is invertible ( $\mathbf{m}$ is a symmetry) if and only if

$$
\mathbf{m}^{*} \mathcal{R}^{0}=\mathcal{R}^{0}, \pi_{0}^{j} \in \mathbf{m}^{*} \Omega \quad(j=1, \ldots, \mu(\Omega)) .
$$

It is however not easy to apply these results effectively in the general equivalence theory. For instance, the solution of the symmetry problem even for the favourable case $\mu(\Omega)=1$ in the famed article [21] was not yet undestood in full detail, see the last sentence in the prominent textbook [22].

\subsection{The Variations [7]}

On the contrary, the surprisingly simple explicit formula

$$
Z=z D+\sum z^{r} \frac{\partial}{\partial t^{r}}+\sum D^{s} p^{j} \frac{\partial}{\partial \pi_{s}^{j}}
$$

for all variations holds true. Here $z, p^{j} \in \mathcal{F}(\mathbf{M})$ are arbitrary functions and $z^{r}=z^{r}\left(t^{1}, \ldots, t^{R}\right)$ arbitrary composed functions. This is a consequence of general Lemma 2.1 applied to the standard basis (30).

\subsection{The Infinitesimal Symmetries $[5],[6],[7]$}

Variation (33) generates a Lie group if and only if it preserves an appropriate good (equivalently: standard) filtration. This is informally described in Figures 1a and 1b where the "dotted filtration" is not known in advance. In the favourable case $\mu(\Omega)=1$, we may deal only with the unique standard filtration 1a and the dotted Figure 1b can be omitted. 


\subsection{A Pesimistic Prediction}

Difficulties appearing in the case $\mu(\Omega)>1$ were explicitly declared in [16]. This is the only pessimistic prognosis for a mathematical theory in E. Cartan's work and it was justified: the higher-order transformations of the absolute theory with the general Monge problem are actually forgotten.

\subsection{The Jet Diffieties}

Passing to more individual examples from now on, we introduce the space $\mathbf{M}(m)$ with local jet coordinates

$$
x, w_{s}^{j} \quad(j=1, \ldots, m ; s=0,1, \ldots)
$$

and the submodule $\Omega(m) \subset \Phi(\mathbf{M}(m))$ of all contact forms

$$
\omega=\sum f_{s}^{j} \omega_{s}^{j} \quad\left(\text { finite sum, } f_{s}^{j} \in \mathcal{F}(\mathbf{M}(m)), \omega_{s}^{j}=\mathrm{d} w_{s}^{j}-w_{s+1}^{j} \mathrm{~d} x\right)
$$

with the total derivative

$$
D=\frac{\partial}{\partial x}+\sum w_{s+1}^{j} \frac{\partial}{\partial w_{s}^{j}} \quad \text { where } \quad \mathcal{L}_{D} \omega_{s}^{j}=\omega_{s+1}^{j}
$$

It follows that we have a diffiety: if $\Omega(m)_{l} \subset \Omega(l=0,1, \ldots)$ is the submodule of the $l$-th order contact forms (with $s \leq l$ in formula (35)) then

$$
\Omega(m)_{*}: \Omega(m)_{0} \subset \Omega(m)_{1} \subset \cdots \subset \Omega=\cup \Omega(m)_{l}
$$

is a good filtration. This is even a standard filtration where $\mathcal{R}^{0}=0$ is trivial module and the contact forms $\omega_{s}^{j}=\pi_{s}^{j}$ provide the standard basis.

We may refer to [2] for many particular examples of automorphisms $\mathbf{m}: \mathbf{M}(m) \rightarrow \mathbf{M}(m)$ of diffiety $\Omega(m)$ involving, e.g., the above wave mechanisms. We recall that they are in general unknown.

On the contrary, all variations

$$
Z=z D+\sum D^{s} p^{j} \frac{\partial}{\partial \omega_{s}^{j}} \quad\left(z, p^{1}, \ldots, p^{m} \text { arbitrary functions }\right)
$$

are well-known. They constitute a huge Lie algebra and one of the main tasks of the soliton theory concerns the determination of the special Abelian Lie subalgebras, the so called integrable hierarchies. On this occasion, we cannot forget the impressive monograph [23]. Variations (37) preserving the filtration (36) are either the infinitesimal point transformations or the infinitesimal Lie's contact transformations. This is a particular case of the Lie-Bäcklund theorem [10],[24]. Variations preserving other good filtrations as in Figure(1b) and therefore generating a group of higher-order transformations are in general unknown.

\subsection{The Hilbert-Cartan Equation}

In order to demonstrate quite explicit results, we mention the infinitesimal symmetries of the ordinary differential equation $d^{2} u / \mathrm{d} x^{2}=F(\mathrm{~d} v / \mathrm{d} x)$ with two unknown functions $u=u(x)$ and $v=v(x)$ thoroughly treated in article [7]. The equation corresponds to the diffiety $\Omega \subset \Phi(\mathbf{M})$ generated by the forms

$$
\alpha_{0}=d u_{0}-u_{1} \mathrm{~d} x, \alpha_{1}=d u_{1}-F\left(v_{1}\right) \mathrm{d} x, \beta_{r}=\mathrm{d} v_{r}-v_{r+1} \mathrm{~d} x \quad(r=0,1, \ldots)
$$

in the space $\mathbf{M}$ with coordinates $x, u_{0}, u_{1}, v_{r}(r=0,1, \ldots)$. Then

$$
D=\frac{\partial}{\partial x}+u_{1} \frac{\partial}{\partial u_{0}}+F \frac{\partial}{\partial u_{1}}+\sum v_{r+1} \frac{\partial}{\partial v_{r}}
$$

where

$$
\left.\mathcal{L}_{D} \alpha_{0}=\alpha_{1}, \mathcal{L}_{D} \alpha_{1}=D\right\rfloor d \alpha_{1}=F^{\prime} \beta_{1}, \mathcal{L}_{D} \beta_{r}=\beta_{r+1} \quad(r=0,1, \ldots)
$$


and the submodules $\Omega_{l} \subset \Omega(l=0,1, \ldots)$ consisting of the forms

$$
\omega=a_{0} \alpha_{0}+a_{1} \alpha_{1}+\sum b_{r} \beta_{r} \quad(r \leq l ; l=0,1, \ldots)
$$

with arbitrary coefficients $a_{0}, a_{1}, b_{r}$ determine the primary good filtration $\Omega_{*}: \Omega_{0} \subset \Omega_{1} \subset \cdots \subset \Omega$ of diffiety $\Omega$. Clearly $\mu(\Omega)=1$, the standard filtration is unique and can be described as follows. Assume $D F^{\prime} \neq 0$ which is satisfied if $F^{\prime \prime} \neq 0$. Abbreviating

$$
\alpha=\alpha_{1}-F^{\prime} \beta_{0}, \pi_{0}^{1}=F^{\prime} \alpha+D F^{\prime} \alpha_{0},
$$

then

$$
\mathcal{L}_{D} \alpha=-D F^{\prime} \beta_{0}, \pi_{1}^{1}=\mathcal{L}_{D} \pi_{0}^{1}=2 D F^{\prime} \alpha+D^{2} F^{\prime} \alpha_{0},
$$

hence $\mathcal{R}^{0}=0$ is the trivial module and the submodules

$$
\begin{aligned}
& \bar{\Omega}_{0}=\left\{\pi_{0}^{1}\right\}=\left\{F^{\prime} \alpha_{1}+D F^{\prime} \alpha_{0}\right\} \\
& \bar{\Omega}_{1}=\left\{\pi_{0}^{1}, \pi_{1}^{1}\right\}=\left\{F^{\prime} \alpha_{1}+D F^{\prime} \alpha_{0}, 2 D F^{\prime} \alpha\right\}=\left\{\alpha, \alpha_{0}\right\} \\
& \bar{\Omega}_{2}=\left\{\alpha, \alpha_{0}, \mathcal{L}_{D} \alpha, \mathcal{L}_{D} \alpha_{0}\right\}=\left\{\alpha, \alpha_{0},-D F^{\prime} \beta_{0}, \alpha_{1}\right\}=\left\{\alpha_{0}, \alpha_{1}, \beta_{0}\right\}=\Omega_{1} \\
& \bar{\Omega}_{3}=\bar{\Omega}_{2}+\mathcal{L}_{D} \bar{\Omega}_{2}=\Omega_{1}+\mathcal{L}_{D} \Omega_{1}=\Omega_{2}, \bar{\Omega}_{4}=\Omega_{3}, \ldots
\end{aligned}
$$

of $\Omega$ constitute the (unique) standard filtration $\bar{\Omega}_{*}$ with the initial form $\pi_{0}^{1}$. The variations

$$
Z=z D+\sum D^{r} p \frac{\partial}{\partial \pi_{r}^{1}} \quad(\text { arbitrary } z, p \in \mathcal{F}(\mathbf{M}(m)))
$$

preserving the standard filtration are just the infinitesimal symmetries. So we have the requirement

$$
\left.\left.\mathcal{L}_{Z} \pi_{0}^{1}=Z\right\rfloor \mathrm{~d} \pi_{0}^{1}+\mathrm{d} \pi_{0}^{1}(Z)=Z\right\rfloor \mathrm{d} \pi_{0}^{1}+\mathrm{d} p=\lambda \pi_{0}^{1}
$$

with unknown factor $\lambda$. The calculations are lengthy. First of all, certain formulae $[7,(107)]$ not stated here uniquely express $z$ and $\lambda$ in terms of the function $p$. Then the crucial equation

$$
p=F^{\prime 2} P(\cdot)+F^{\prime} Q(\cdot) v_{2} \quad\left((\cdot)=\left(x, u_{0}, u_{1}, v_{0}, v_{1}\right)\right)
$$

where

$$
P=\frac{1}{F^{\prime}}\left(Q_{x}+u_{1} Q_{u_{0}}\right)+\int\left(\frac{1}{F^{\prime}}\right)^{\prime} F \mathrm{~d} v_{1} \cdot Q_{u_{1}}+\int\left(\frac{1}{F^{\prime}}\right)^{\prime} v_{1} \mathrm{~d} v_{1} \cdot Q_{v_{0}}+\bar{P}\left(x, u_{0}, u_{1}, v_{0}\right)
$$

can be derived, see $[7,(133)]$. In the generic subcase $[7,(144)]$ we obtain the final solution

$$
Q=(A x+\bar{A}) u_{1}+B v_{0}+C_{1} x-2 A u_{0}+C_{3}, \bar{P}=A v_{0}+C \quad(A, \ldots, C \in \mathbb{R})
$$

but special functions $F$ admit more symmetries. For instance the exceptional 14-dimensional Lie algebra $\mathbb{G}_{2}$ of symmetries for the Hilbert-Cartan equation where $F=(\mathrm{d} v / \mathrm{d} x)^{1 / 2}$ was obtained $[7,(175)]$ by for the first time direct calculations in full accordance with quite other approach in the article [21].

\subsection{Example: the Monge Equation}

We mention the differential equation $\mathrm{d} w / \mathrm{d} x=F(d u / \mathrm{d} x, \mathrm{~d} v / \mathrm{d} x)$ with nonconstant $F$, however, only some conceptual topics will be discussed and we refer to [7] for more detailed survey. The corresponding diffiety $\Omega \subset \Phi(\mathbf{M})$ has the natural basis denoted

$$
\alpha_{r}=d u_{r}-u_{r+1} \mathrm{~d} x, \beta_{r}=\mathrm{d} v_{r}-v_{r+1} \mathrm{~d} x \quad(r=0,1, \ldots), \gamma=\mathrm{d} w-F\left(u_{1}, v_{1}\right) \mathrm{d} x
$$

in the space $\mathbf{M}$ with coordinates $x, u_{r}, v_{r}(r=0,1, \ldots)$ and $w$. The forms $\alpha_{r}, \beta_{r}(r \leq l)$ and $\gamma$ constitute a basis of module $\Omega_{l}(l=0,1, \ldots)$ of the original good filtration $\Omega_{*}$. The obvious identity

$$
\mathcal{L}_{D} \gamma=F_{u_{1}} \alpha_{1}+F_{v_{1}} \beta_{1} \quad\left(D=\frac{\partial}{\partial x}+\sum u_{r+1} \frac{\partial}{\partial u_{r}}+\sum v_{r+1} \frac{\partial}{\partial v_{r}}+F \frac{\partial}{\partial w} \in \mathcal{H}\right)
$$


represents the "cross" in Figure 2a. Then $\mathcal{R}^{0}=0$ and the forms

$$
\pi_{0}^{1}=\gamma-F_{u_{1}} \alpha_{0}-F_{v_{1}} \beta_{0}, \pi_{0}^{2}=\beta_{0}
$$

are initial for the standard filtration of Figure 2b. In more detail, the formulae

$$
\mathcal{L}_{D} \alpha_{r}=\alpha_{r+1}, \mathcal{L}_{D} \beta_{r}=\beta_{r+1} \quad(r=0,1, \ldots), \mathcal{L}_{D} \gamma=F_{u_{1}} \alpha_{1}+F_{v_{1}} \beta_{1}
$$

imply

$$
\pi_{1}^{1}=\mathcal{L}_{D} \pi_{0}^{1}=-D F_{u_{1}} \alpha_{0}-D F_{v_{1}} \beta_{0}
$$

whence the submodules

$$
\begin{aligned}
& \bar{\Omega}_{0}=\left\{\pi_{0}^{1}, \pi_{0}^{2}\right\}=\left\{\gamma-F_{u_{1}} \alpha_{0}, \beta_{0}\right\} \\
& \bar{\Omega}_{1}=\bar{\Omega}_{0}+\mathcal{L}_{D} \bar{\Omega}_{0}=\left\{\pi_{0}^{1}, \pi_{0}^{2}, \pi_{1}^{1}, \pi_{1}^{2}\right\}=\left\{\alpha_{0}, \beta_{0}, \gamma\right\}=\Omega_{0}, \\
& \bar{\Omega}_{2}=\bar{\Omega}_{1}+\mathcal{L}_{D} \bar{\Omega}_{1}=\Omega_{1}, \bar{\Omega}_{3}=\Omega_{2}, \ldots
\end{aligned}
$$

of module $\Omega$ constitute a good filtration. The alternative basis

$$
\pi_{0}^{1}=\gamma+\cdots, \pi_{1}^{1}=-D F_{u_{1}} \alpha_{0}+\cdots, \pi_{2}^{1}=-D F_{u_{1}} \alpha_{1}+\cdots, \cdots, \pi_{r}^{2}=\beta_{r}(r \geq 0)
$$

of diffiety $\Omega$ is better adapted for the calculations than the original one thanks to the absence of "crossings".

Let us start with morphisms $\mathbf{m}: \mathbf{M} \rightarrow \mathbf{M}$ of diffiety $\Omega$. They are determined by formulae

$$
\mathbf{m}^{*} \pi_{0}^{1}=\sum a_{r}^{j} \pi_{r}^{j}, \mathbf{m}^{*} \pi_{0}^{2}=\sum b_{r}^{j} \pi_{r}^{j} \quad \text { (arbitrary coefficients) }
$$

since the remaining forms

$$
\mathbf{m}^{*} \pi_{r}^{k}=\mathbf{m}^{*} \mathcal{L}_{D}^{r} \pi_{0}^{k} \quad(k=1,2 ; r=1,2, \ldots)
$$

satisfy the recurrence (31). We are however interested in invertible morphisms $\mathbf{m}$ though the criterion (32) provides rather strong additional condition for the coefficients $a_{r}^{j}$ and $b_{r}^{j}$. The problem is not yet completely resolved.

On the contrary, all variations

$$
Z=z D+\sum D^{r} p \frac{\partial}{\partial \pi_{r}^{1}}+\sum D^{r} q \frac{\partial}{\partial \pi_{r}^{2}} \quad(\operatorname{arbitrary} z, p, q)
$$

are given by simple explicit formulae. We are however interested also in infinitesimal symmetries $Z$ and they moreover are bound to preserve a certain good filtration (Figures 1a and $1 \mathrm{~b}$ ). Since $\mu(\Omega)=2$, there are many possibilities and this is the reason why the symmetry problem for our seemingly simple Monge equation is extremely difficult. We can only refer to three particular examples of symmetries

$$
\begin{aligned}
& \mathcal{L}_{Z} \pi_{0}^{1}=\mu \pi_{0}^{1}, \mathcal{L}_{Z} \pi_{0}^{2}=\lambda_{0}^{1} \pi_{0}^{1}+\lambda_{0}^{2} \pi_{0}^{2}+\lambda_{1}^{1} \pi_{1}^{1}, \\
& \mathcal{L}_{Z} \pi_{0}^{1}=\mu_{0}^{1} \pi_{0}^{1}+\mu_{0}^{2} \pi_{0}^{2}+\mu_{1}^{2} \pi_{1}^{2}, \mathcal{L}_{Z} \pi_{0}^{2}=\mu \pi_{0}^{2}, \\
& \mathcal{L}_{Z} \pi_{0}^{1}=\lambda^{1} \pi_{0}^{1}+\lambda^{2} \pi_{0}^{2}, \mathcal{L}_{Z} \pi_{0}^{2}=\mu^{1} \pi_{0}^{1}+\mu^{2} \pi_{0}^{2}
\end{aligned}
$$

discussed in [7] especially for the case $F=u_{1} v_{1}$.

\subsection{The Monge Problem}

There exists an automorphism $\mathbf{m}: \mathbf{M}(3) \rightarrow \mathbf{M}(3)$ of the jet diffiety $\Omega(3)$ such that

$$
\mathbf{m}^{*}\left(x-F\left(w_{0}^{1}, w_{0}^{2}\right)\right)=w_{1}^{3}-F\left(w_{1}^{1}, w_{1}^{2}\right)
$$

for every nonconstant function $F$, see [7, Appendix] and this may be regarded as a solution of a Monge problem, which is as follows. 
We recall coordinates $x, w_{s}^{j}(j=1,2,3 ; s=0,1, \ldots)$ of the space $\mathbf{M}(3)$ and the diffiety $\Omega(3)$ generated by all contact forms $\omega_{s}^{j}=\mathrm{d} w_{s}^{j}-w_{s+1}^{j} \mathrm{~d} x$ where the solutions are given by formulae

$$
w_{s}^{j}=w_{s}^{j}(x)=\frac{\mathrm{d}^{s} w^{j}(x)}{\mathrm{d} x^{s}} \quad\left(\text { arbitrary functions } w^{j}(x) ; j=1,2,3\right) .
$$

(In traditional terms, we deal with all curves in four-dimensional space with variables $x, w^{1}, w^{2}, w^{3}$.) Let us consider only such curves that moreover satisfy

$$
x=F\left(w^{1}(x), w^{2}(x)\right) .
$$

(Informally, they are lying in the three-dimensional subspace $x=F\left(w^{1}, w^{2}\right)$. In fact we have the lower diffiety $\Omega(2)$.) The mentioned automorphism $\mathbf{m}$ identifies these curves with all solutions of the Monge equation

$$
\frac{\mathrm{d} w_{0}^{3}}{\mathrm{~d} x}=F\left(\frac{\mathrm{d} w_{0}^{1}}{\mathrm{~d} x}, \frac{\mathrm{d} w_{0}^{2}}{\mathrm{~d} x}\right) .
$$

(Altogether, there is explicit correspondence between solutions of diffiety $\Omega(2)$ and solutions of the Monge equation.)

Still, in other terms, we have the subspace $\mathbf{N} \subset \mathbf{M}(3)$ given by equations

$$
D^{r}\left(x-F\left(w_{0}^{1}, w_{0}^{2}\right)\right)=0 \quad\left(r=0,1, \ldots ; D=\frac{\partial}{\partial x}+\sum w_{r+1}^{j} \frac{\partial}{\partial w_{r}^{j}}\right)
$$

which is clearly isomorphic to $\mathbf{M}(2)$. It follows that the corresponding jet diffiety $\Omega(2) \subset \Phi(\mathbf{M}(2))$ is isomorphic to the diffiety $\Omega$ of the Monge equation. Roughly saying, the diffiety $\Omega$ of the Monge equation is in fact the "trivial" diffiety $\Omega(2)$.

\subsection{Calculus of Variations [5],[7],[25],[26],[27],[28]}

The classical Lagrange problem concerning the one-dimensional variational integral subject to differential constraints is represented by a diffiety $\Omega \subset \Phi(\mathbf{M})$ together with a given form $\varphi \in \Phi(\mathbf{M})$. We are interested in the variational integrals

$$
\int_{a}^{b} \mathbf{n}^{*} \varphi \quad\left(\mathbf{n}: \mathbf{N} \rightarrow \mathbf{M}, \mathbf{n}^{*} \Omega=0, \mathbf{N}: a \leq x \leq b \subset \mathbb{R}\right)
$$

evaluated for the solutions $\mathbf{n}: \mathbf{N} \rightarrow \mathbf{M}$ of diffiety $\Omega$. Such a solution is called extremal if $\left.\mathbf{n}^{*} Z\right\rfloor d \varphi=0$ for all variations $Z$ along $\mathbf{N}$, that is, $\mathbf{n}^{*} \mathcal{L}_{Z} \Omega=0$. This is in full accordance with the common approach since then the obvious identities

$$
\mathbf{n}^{*} \mathcal{L}_{Z} \varphi=\operatorname{d} \mathbf{n}^{*} \varphi(Z), \int_{a}^{b} \mathbf{n}^{*} \mathcal{L}_{Z} \varphi=\left.\mathbf{n}^{*} \varphi(Z)\right|_{x=a} ^{x=b}
$$

declare that the variation of the integral (in the common sense) indeed depends only on the boundary values. One can observe that the form $\varphi$ can be replaced with any form $\varphi+\omega(\omega \in \Omega)$ without change of the extremals. At this place, let us apply the standard filtrations. Assuming the controllability $\mathcal{R}^{0}=0$, a unique Poincaré-Cartan form $\breve{\varphi}=\varphi+\breve{\omega}$ with appropriate $\breve{\omega} \in \Omega$ exists such that

$$
\mathrm{d} \breve{\varphi} \cong \sum e^{j} \pi_{0}^{j} \wedge \mathrm{d} x \quad(\bmod \Omega \wedge \Omega ; j=1, \ldots, \mu(\Omega))
$$

This implies that the extremals $\mathbf{n}$ are characterized by the Euler-Lagrange equations $\mathbf{n}^{*} e^{j}=0(j=$ $1, \ldots, \mu(\Omega))$ and satisfy the identities

$$
\left.\mathbf{n}^{*} Z\right\rfloor \mathrm{d} \breve{\varphi}=0, \int_{a}^{b} \mathbf{n}^{*} \mathcal{L}_{Z} \breve{\varphi}=\left.\mathbf{n}^{*} \breve{\varphi}(Z)\right|_{x=a} ^{x=b}
$$

for all vector fields $Z \in \mathcal{T}(\mathbf{M})$. 
In particular $e^{j}=0(j=1,2, \ldots)$ identically if and only if the Poincaré-Cartan form $\breve{\varphi}$ is a total differential of appropriate function $g$ hence $\varphi \cong \breve{\varphi} \cong D g \cdot \mathrm{d} x(\bmod \Omega)$. The Noether theorem immediately follows as well. Assuming

$$
\left.\mathcal{L}_{Z} \Omega \subset \Omega, \mathcal{L}_{Z} \varphi \in \Omega \quad \text { (appropriate } Z \in \mathcal{T}(\mathbf{M})\right),
$$

the function $\left.\mathbf{n}^{*} Z\right\rfloor \mathrm{d} \breve{\varphi}$ is clearly constant for every extremal $\mathbf{n}$.

\subsection{Example: a Variational Integral}

We mention only the variational integral

$$
\int f\left(x, u_{0}, v_{0}, w_{0}, u_{1}, v_{1}\right) \mathrm{d} x \quad\left(u_{r}=\frac{\mathrm{d}^{r} u}{\mathrm{~d} x^{r}}, v_{r}=\frac{\mathrm{d}^{r} v}{\mathrm{~d} x^{r}}\right)
$$

with the constraint

$$
w_{1}=F\left(x, u_{0}, v_{0}, w_{0}, u_{1}, v_{1}\right)
$$

Assuming

$$
a=F_{v_{0}}-D F_{v_{1}}+F_{w_{0}} F_{v_{1}} \neq 0 \quad\left(D=\frac{\partial}{\partial x}+\sum u_{r+1} \frac{\partial}{\partial u_{r}}+\sum v_{r+1} \frac{\partial}{\partial v_{r}}+F \frac{\partial}{\partial w_{0}}\right),
$$

there exists the Poincaré-Cartan form

$$
\breve{\varphi}=f \mathrm{~d} x+\left(f_{u_{1}}-\frac{b}{a} F_{u_{1}}\right) \alpha+\left(f_{v_{1}}-\frac{b}{a} F_{v_{1}}\right) \beta-\frac{b}{a} \gamma
$$

where

$$
b=f_{v_{0}}-D f_{v_{1}}+f_{w_{0}} F_{v_{1}}, \alpha=d u_{0}-u_{1} \mathrm{~d} x, \beta=\mathrm{d} v_{0}-v_{1} \mathrm{~d} x, \gamma=\mathrm{d} w_{0}-F \mathrm{~d} x
$$

and the Euler-Lagrange equations

$$
e^{1}=f_{w_{0}}-\frac{b}{a} F_{w_{0}}-D \frac{b}{a}=0, e^{2}=B-\frac{b}{a} A=0
$$

where

$$
A=F_{u_{0}}-D F_{u_{1}}+F_{w_{0}} F_{u_{1}}, B=f_{u_{0}}-D f_{u_{1}}+f_{w_{0}} F_{u_{1}} .
$$

Since no uncertain multipliers appear, the Legendre, Jacobi, Hilbert-Weierstrass extremality conditions, the Hamilton-Jacobi equations and the geodesic fields can be investigated without much difficulty quite analogously as in the traditional unconstrained theory [25],[26],[27],[28].

Acknowledgments. This paper was elaborated with the financial support of the European Union's "Operational Programme Research and Development for Innovations", No. CZ.1.05/2.1.00/03.0097, as an activity of the regional Centre AdMaS Operational Programme Research and Development for "Advanced Materials, Structures and Technologies".

\section{References}

1. Veronika Chrastinová and Václav Tryhuk. Automorphisms of submanifolds. Adv. Difference Equ., pages Art. ID 202731, 26, 2010.

2. Václav Tryhuk and Veronika Chrastinová. Automorphisms of curves. J. Nonlinear Math. Phys., 16(3):259-281, 2009.

3. Veronika Chrastinová. Parallel curves in three-dimensional space. In 5-th Conference on Mathematics and Physics on Technical Universities, pages 120-124. University of Defence, Brno, 2007.

4. Veronika Chrastinová and Václav Tryhuk. Generalized contact transformations. Journal of Applied Mathematics, Statistics and Informatics, 3(1):47-62, 2007.

5. Jan Chrastina. The formal theory of differential equations, volume 6 of Folia Facultatis Scientiarium Naturalium Universitatis Masarykianae Brunensis. Mathematica. Masaryk University, Brno, 1998. 
6. V. Tryhuk, V. Chrastinová, and O. Dlouhý. The Lie group in infinite dimension. Abstr. Appl. Anal., pages Art. ID 919538, 35, 2011.

7. Václav Tryhuk and Veronika Chrastinová. Automorphisms of ordinary differential equations. Abstr. Appl. Anal., pages Art. ID 482963, 32, 2014.

8. Veronika Chrastinová and Václav Tryhuk. On the internal approach to differential equations 1 . The involutiveness and standard basis. Math. Slovaca, 66(4):999-1018, 2016. DOI: 10.1515/ms-2015-0198.

9. Veronika Chrastinová and Václav Tryhuk. On the internal approach to differential equations 2. The controllability structure. Math. Slovaca, 2014. (arXiv:1409.5904) accepted.

10. Veronika Chrastinová and Václav Tryhuk. On the internal approach to differential equations 3. Infinitesimal symmetries. Math. Slovaca, 2014. (arXiv:1403.0781) accepted.

11. R. L. Bryant, S. S. Chern, R. B. Gardner, H. L. Goldschmidt, and P. A. Griffiths. Exterior differential systems, volume 18 of Mathematical Sciences Research Institute Publications. Springer-Verlag, New York, 1991.

12. I. S. Krasil'shchik, V. V. Lychagin, and A. M. Vinogradov. Geometry of jet spaces and nonlinear partial differential equations, volume 1 of Advanced Studies in Contemporary Mathematics. Gordon and Breach Science Publishers, New York, 1986. Translated from the Russian by A. B. Sosinskiu.

13. A. M. Vinogradov. Cohomological analysis of partial differential equations and secondary calculus, volume 204 of Translations of Mathematical Monographs. American Mathematical Society, Providence, RI, 2001. Translated from the Russian manuscript by Joseph Krasil'shchik.

14. Ch. Riquier. Les systèmes d'équations aux dérivées partielles. Gauthier-Villars, 1910.

15. Élie Cartan. L'intégration des systèmes d'équations aux différentielles totales. Ann. Sci. École Norm. Sup. (3), 18:241-311, 1901.

16. Élie Cartan. Les sous-groupes des groupes continus de transformations. Ann. Sci. Éc. Norm. Supér. (3), 25:57-194, 1908.

17. Élie Cartan. Les systèmes différentiels exterieurs et leurs applications geométriques. Paris: Hermann \& Cie. 214 p. (1945)., 1945.

18. Bernard Malgrange. Cartan involutiveness = Mumford regularity. In Commutative algebra (Grenoble/Lyon, 2001), volume 331 of Contemp. Math., pages 193-205. Amer. Math. Soc., Providence, RI, 2003.

19. Élie Cartan. Sur l'équivalence absolue de certains systèmes d'équations différentielles et sur certaines familles de courbes. Bull. Soc. Math. France, 42:12-48, 1914.

20. David Hilbert. Über den begriff der klasse von differentialgleichungen. Math. Ann., 73(1):95-108, 1912.

21. Elie Cartan. Les systèmes de Pfaff, à cinq variables et les équations aux dérivées partielles du second ordre. Ann. Sci. École Norm. Sup. (3), 27:109-192, 1910.

22. Shlomo Sternberg. Lectures on differential geometry. Prentice-Hall, Inc., Englewood Cliffs, N.J., 1964.

23. Boris A. Kupershmidt. KP or $m K P$, volume 78 of Mathematical Surveys and Monographs. American Mathematical Society, Providence, RI, 2000. Noncommutative mathematics of Lagrangian, Hamiltonian, and integrable systems.

24. Václav Tryhuk and Veronika Chrastinová. On the mapping of jet spaces. J. Nonlinear Math. Phys., 17(3):293-310, 2010.

25. Jan Chrastina. Examples from the calculus of variations. I. Nondegenerate problems. Math. Bohem., 125(1):55-76, 2000.

26. Jan Chrastina. Examples from the calculus of variations. II. A degenerate problem. Math. Bohem., 125(2):187197, 2000.

27. Jan Chrastina. Examples from the calculus of variations. III. Legendre and Jacobi conditions. Math. Bohem., 126(1):93-111, 2001.

28. Jan Chrastina. Examples from the calculus of variations. IV. Concluding review. Math. Bohem., 126(4):691-710, 2001. 